\title{
Chiral Koszul Duality
}

\section{Citation}

Francis, John, and Dennis Gaitsgory. 2012. Chiral Koszul duality. Selecta Mathematica, n.s., 18(1): 27-87.

\section{Published Version}

doi:10.1007/s00029-011-0065-z

\section{Permanent link}

http://nrs.harvard.edu/urn-3:HUL.InstRepos:10043337

\section{Terms of Use}

This article was downloaded from Harvard University's DASH repository, and is made available under the terms and conditions applicable to Open Access Policy Articles, as set forth at http:// nrs.harvard.edu/urn-3:HUL.InstRepos:dash.current.terms-of-use\#OAP

\section{Share Your Story}

The Harvard community has made this article openly available.

Please share how this access benefits you. Submit a story.

Accessibility 


\title{
CHIRAL KOSZUL DUALITY
}

\author{
JOHN FRANCIS AND DENNIS GAITSGORY
}

\begin{abstract}
We extend the theory of chiral and factorization algebras, developed for curves by Beilinson and Drinfeld in BD1, to higher-dimensional varieties. This extension entails the development of the homotopy theory of chiral and factorization structures, in a sense analogous to Quillen's homotopy theory of differential graded Lie algebras. We prove the equivalence of higherdimensional chiral and factorization algebras by embedding factorization algebras into a larger category of chiral commutative coalgebras, then realizing this interrelation as a chiral form of Koszul duality. We apply these techniques to rederive some fundamental results of [BD1] on chiral enveloping algebras of $\star$-Lie algebras.
\end{abstract}

\section{Contents}

1. Introduction

1.1. Why study chiral algebras?

1.2. Contents

1.3. $\infty$-categories

1.4. D-modules

1.5. Acknowledgements

2. Chiral algebras and factorization coalgebras

2.1. D-modules on the Ran space

2.2. Symmetric monoidal structures on $\mathfrak{D}(\operatorname{Ran} X)$

2.3. Explicit description of tensor product functors

2.4. Chiral Lie algebras and factorization coalgebras

2.5. Variant: the topological context

3. Algebras and coalgebras over (co)operads: recollections

3.1. Operads

3.2. Algebras over an operad

3.3. Koszul duality functors

3.4. Turning Koszul duality into an equivalence

3.5. Coalgebras over an operad

4. Koszul duality in nilpotent tensor $\infty$-categories

4.1. Nilpotent and pro-nilpotent tensor $\infty$-categories

4.2. Coalgebras vs. ind-nilpotent coalgebras in the pro-nilpotent case

4.3. The case of Lie algebras

5. Proof of the main theorem

5.1. Koszul duality in the chiral setting

5.2. Factorization

6. Chiral envelopes of $\star$-Lie algebras

6.1. The basic commutative diagram

6.2. Recollections on monads 31

6.3. Chiral homology of chiral envelopes

6.4. Chiral envelopes and factorization 34

2010 Mathematics Subject Classification. Primary 81R99. Secondary 14H81, 18 G55.

Key words and phrases. Chiral algebras. Chiral homology. Factorization algebras. Conformal field theory. Koszul duality. Operads. $\infty$-Categories. 
6.5. The Poincaré-Birkhoff-Witt theorem

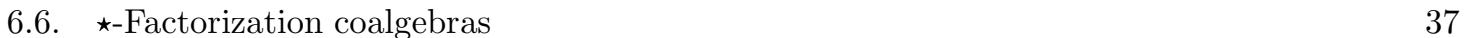

7. Chiral and factorization modules $\quad 38$

7.1. Modules for algebras over an operad $\quad 38$

\begin{tabular}{|l|l|} 
7.2. Chiral and $\star$ actions & 39 \\
\hline
\end{tabular}

7.3. Chiral and factorization modules $\quad 41$

References

\section{INTRODUCTION}

Beilinson and Drinfeld developed the theory of chiral and factorization (co)algebras on curves in their seminal work, BD1, as a geometric counterpart of the algebraic theory of vertex algebras. Their theory translated the formulae of operator product expansions in conformal field theory into beautiful algebraic geometry. These two algebraic avatars of conformal field theory at first blush appear quite dissimilar: A chiral Lie algebra is a D-module on a curve with a type of Lie algebra structure in which one has the extra ability to take the Lie bracket of certain divergent sections; a factorization coalgebra consists of a quasi-coherent sheaf on each configuration space of a curve, with certain compatibilities. One of the conceptually central results of [BD1] (Theorem 3.4.9) establishes the equivalence of these two theories of chiral Lie algebras and of factorization coalgebras on algebraic curves.

Beilinson and Drinfeld posed several challenges left open by their work: first, to extend their theory above complex dimension 1 . Second, in order to sensibly extend the theory to varieties, they observed the necessity of developing the homotopy theory of chiral Lie algebras (in a sense analogous to Quillen's homotopy theory of differential graded algebras), a problem of independent interest 1

In this work, we develop just such a homotopy theory of chiral and factorization structures and apply it to prove a generalization of the above theorem of [BD1], to establish an equivalence between chiral Lie algebras and factorization coalgebras on higher-dimensional varieties. The most appealing aspect of this proof is a reconceptualization of the relation between the two: The equivalence between chiral Lie algebras and factorization coalgebras is a form of Koszul duality, in which factorization coalgebras are realized as a full subcategory of a larger category of chiral commutative coalgebras 2 This is a chiral analogue of the duality between Lie algebras and commutative coalgebras that Quillen first developed in his work on rational homotopy theory, Q2, in which the category of chain complexes, with tensor product, is replaced by that of D-modules on the Ran space, equipped with the chiral tensor product of D-modules. We shall see that despite this apparent increased complexity, chiral Koszul duality is more of a duality than usual Koszul duality, in the sense that the double dual is always a homotopy equivalence, without preconditions.

Beilinson and Drinfeld's perspective on chiral versus factorization gave rise to an important new construction, the chiral homology of chiral Lie algebras, a homotopy-theoretic generalization of the space of conformal blocks in conformal field theory. The other primary focus of [BD1] was the calculation of chiral homology in several salient examples, including lattice chiral Lie algebras and chiral enveloping algebras of $\star$-Lie algebras. Chiral enveloping algebras are chiral analogues of the usual enveloping algebra of a Lie algebra; they appear in conformal field theory in the construction of affine Kac-Moody chiral Lie algebras, and as such serve as chiral versions of the Lie algebras of loop groups. To illustrate the efficacy of the Koszul duality viewpoint, as an application we give

\footnotetext{
${ }^{1}$ For instance, the category of chiral Lie algebras on a curve $X$ lacks coproducts, hence it cannot admit a model category structure.

${ }^{2}$ It is for this reason that we take the liberty of adjusting the terminology "factorization algebra" of BD1 to "factorization coalgebra," since they are, literally, coalgebras rather than algebras with respect to the chiral tensor structure. See Remark 2.4 .8
} 
a conceptual proof of Theorem 4.8.1.1 of [BD1], which expresses the chiral homology of the chiral envelope of a $\star$-Lie algebra $L$ in terms of de Rham cohomology of $L$ itself.

1.1. Why study chiral algebras? Before giving an overview of the contents of this paper, let us offer some general motivation for the study of chiral Lie algebras and factorization coalgebras. Broadly speaking, one can divide the reasons to study them into two classes: local and global.

1.1.1. Locally, chiral Lie algebras and their representations on curves appear as a general formalism to study representation theory of Lie algebras that have a loop component, as well as categories obtained from the category of Lie algebra modules by various functorial procedures.

For example, consider the Lie algebra of formal Laurent series $\mathfrak{g}((t))$, where $\mathfrak{g}$ is a finite-dimensional Lie algebra. There is a direct route to studying representations of $\mathfrak{g}(t))$, but in which one is required to take into account the topology on $\mathfrak{g}((t))$ : This is certainly doable, though it makes homological algebra more cumbersome. However, to then further study those representations that are integrable (i.e., those that arise from differentiating positive energy representation of the loop group $G((t))$ ), becomes impracticable from the vantage of topological associative algebras.

Another local aspect of the story is the connection between chiral Lie algebras and $\mathcal{E}_{2}$-algebras. Via the Riemann-Hilbert correspondence, $\mathcal{E}_{2}$-algebras form a full subcategory of chiral Lie algebras on the affine line, consisting of those chiral algebras whose underlying D-module is holonomic with regular singularities.

This perspective allowed one to rediscover chiral Lie algebras in their factorization incarnation in the work of Schechtman-Varchenko and its elaboration by Bezrukavnikov-Finkelberg-Schechtman (see $\mathrm{BFS}$ ] and references therein) on the construction of quantum groups via configuration spaces, and its relation to the Kazhdan-Lusztig equivalence between quantum groups and Kac-Moody representations.

Further, the discovery of factorization coalgebras led to the notion of a factorization category, which appears as a very potent tool for many problems of geometric representation theory (see Ga1] for a brief review).

1.1.2. Let us now turn to the global aspects of the theory. For this discussion we assume that $X$ is complete. The overarching reason for the usefulness of chiral Lie algebras is that the procedure of taking chiral homology of chiral Lie algebras/factorization coalgebras is a powerful local-to-global principle.

For example, let $Y$ be a scheme affine over $X$, and suppose one is interested to study the scheme of its global sections $X \rightarrow Y$. According to BD1, Theorem 4.6.1, this scheme can be described as Spec of the chiral homology of a certain chiral algebra.

The above example is "commutative" in the sense of BD1, Sect. 4.6. A non-commutative, but relatively elementary, example of an application of the above local-to-global principle is the construction of Hecke eigensheaves in the geometric Langlands program carried out in BD2].

However, this local-to-global principle can be applied in significantly more sophisticated situations. In particular, it plays a prominent role in the recent advances in the geometric Langlands program, where one applies it in the case of chiral Lie algebra that controls twisted Whittaker sheaves.

We should also remark that the functor of chiral homology on the category of chiral Lie algebras bears a strong similarity with the assignment in quantum field theory to a collection of local observables of the value of the corresponding correlation function at a particular configuration of points on a compact space-time. 
1.2. Contents. We now review the contents of the paper and state our main results:

Throughout the paper we will be working over a ground field $k$ of characteristic 0 . We will be working with the category Sch of schemes of finite type over $k$, and for any $Y \in$ Sch we denote by $\mathfrak{D}(Y)$ the stable $\infty$-category of D-modules on $Y$ (see Section 1.4.1 where our conventions regarding $\mathfrak{D}(Y)$ are explained).

For the duration of the paper we fix $X$ to be a separated scheme of finite type over $k$.

1.2.1. The Ran space. Our main geometric object of study is the Ran space of $X$, which should be thought of as the "space of all finite configurations of points of $X$," and the category of D-modules on it. In other words, $\operatorname{Ran} X$ is intuitively given by the union $\bigcup_{j} \operatorname{Conf}_{j} X$ of the configuration spaces of unordered points in $X$, topologized so that that two points may collide and pass to a different stratum, i.e., so that the map $X^{I} \rightarrow \bigcup_{j} \operatorname{Conf}_{j} X$ is continuous for each $n$. However, this intuition does not immediately translate into a genuine definition: $\operatorname{Ran} X$ does not exist as a scheme or even an ind-scheme, and so the category of D-modules on it is not a priori defined.

To remedy this, we can consider the structure that we would see if $\operatorname{Ran} X$ did exist as described: For a D-module $M$ on $\operatorname{Ran} X$, we could pull it back to $X^{I}$ to obtain a new D-module, $M^{I}$, for each finite set $I$; these D-modules would be subject to certain compatibilities under pullbacks, given a factorization $X^{J} \rightarrow X^{I} \rightarrow \operatorname{Ran} X$. One should imagine that you can completely recover the D-module $M$ from this compatible family of $M^{I}$.

This intuition gives rise to a formal definition. We define $\operatorname{Ran} X$ as a functor from the category opposite that of finite sets to Sch, namely $I \leadsto X^{I}$, and define the $\infty$-category $\mathfrak{D}(\operatorname{Ran} X)$ as the limit of $\mathfrak{D}\left(X^{I}\right)$ over finite maps. I.e., an object $M \in \mathfrak{D}(\operatorname{Ran} X)$ is by definition a collection of objects $M^{I} \in \mathfrak{D}\left(X^{I}\right)$ for each finite set $I$ and a homotopy equivalence

$$
\Delta(\pi)^{!}\left(M^{I}\right) \simeq M^{J}
$$

for every surjection $\pi: I \rightarrow J$, where $\Delta^{\pi}: X^{J} \rightarrow X^{I}$ denotes the corresponding map.

Now, following [BD1] we introduce two symmetric monoidal structures on $\mathfrak{D}(\operatorname{Ran} X)$. The first one, the $\star$-tensor product, should be thought of as the direct image with respect to the map

$$
\text { union : } \operatorname{Ran} X \times \operatorname{Ran} X \longrightarrow \operatorname{Ran} X
$$

given by the operation of union of finite sets. I.e., it is convolution with respect to the abelian semi-group structure on $\operatorname{Ran} X$.

The other symmetric monoidal structure, the chiral tensor product, is the composition

$$
\operatorname{union}_{*} \circ \jmath_{*} \circ \jmath^{*}
$$

where $\jmath$ is the open embedding of the locus

$$
(\operatorname{Ran} X \times \operatorname{Ran} X)_{\text {disj }} \subset \operatorname{Ran} X \times \operatorname{Ran} X,
$$

corresponding to pairs of finite subsets of $X$ that are disjoint.

In other words, one should think about these two tensor products as follows. For $M_{1}, M_{2} \in$ $\mathfrak{D}(\operatorname{Ran} X)$, the fiber of $M_{1} \otimes^{\star} M_{2}$ (resp., $M_{1} \otimes^{\text {ch }} M_{2}$ ) at a point $\{S\} \in \operatorname{Ran} X$, where $S \subset X$ is a finite non-empty subset is

$$
\underset{S=S_{1} \cup S_{2}}{\oplus}\left(M_{1}\right)_{S_{1}} \otimes\left(M_{2}\right)_{S_{2}},
$$

where for the $\star$-tensor product the direct sum is taken over all decompositions as a union of nonempty subsets, and for the chiral tensor product we only take those summands for which $S_{1} \cap S_{2}=\varnothing$. 
1.2.2. Chiral algebras and factorization coalgebras. Consider the $\infty$-category $\mathfrak{D}(\operatorname{Ran} X)$, endowed with the chiral tensor structure. We can consider the categories of Lie algebras and commutative coalgebras in it, denoted Lie-alg ${ }^{\mathrm{ch}}(\operatorname{Ran} X)$ and Com-coalg ${ }^{\text {ch }}(\operatorname{Ran} X)$, respectively.

Inside Lie-alg ${ }^{\text {ch }}(\operatorname{Ran} X)$ we single out the full subcategory spanned by objects that, as Dmodules on $\operatorname{Ran} X$, are supported on the main diagonal $X \subset \operatorname{Ran} X$. We denote this $\infty$-category by Lie-alg ${ }^{\mathrm{ch}}(X)$. This is the $\infty$-category of chiral Lie algebras introduced by [BD1].

Inside Com-coalg ${ }^{\text {ch }}(\operatorname{Ran} X)$ we single out a full subcategory of factorization coalgebras that we denote by Com-coalg ${ }_{\text {Fact }}^{\text {ch }}(\operatorname{Ran} X)$. We shall also use the notation Fact $(X)$ and refer to the objects of this $\infty$-category as factorization D-modules on $\operatorname{Ran} X$. We shall now indicate its definition:

Let $B$ be a coalgebra in $\mathfrak{D}(\operatorname{Ran} X)$. Let $S \subset X$ be a finite subset, and $S=S_{1} \sqcup S_{2}$ be its decomposition as a disjoint union. Then the coalgebra structure on $B$ defines a map at the level of fibers

$$
B_{S} \longrightarrow B_{S_{1}} \otimes B_{S_{2}}
$$

The factorizability condition is that the above map should be a homotopy equivalence.

Note that the notion of factorization coalgebra can be encoded as an assignment

$$
(S \subset X) \leadsto\left(B_{S} \in \operatorname{Vect}_{k}\right),
$$

(and such that this system forms a D-module as $S$ ranges over $\operatorname{Ran} X$ ), and a system of homotopy equivalences (1.1) that satisfy the natural compatibility conditions under further partitions of finite sets into disjoint unions. When written in this form, the notion of factorization D-module looks symmetric from the algebra/coalgebra perspective.

1.2.3. Koszul duality. Let us now recall the following general construction. Let $\mathcal{C}$ be a (not necessarily unital) stable symmetric monoidal $\infty$-category over $k$. We can consider the $\infty$-category $\operatorname{Lie}-\operatorname{alg}(\mathcal{C})$ of Lie algebras in $\mathcal{C}$, and the $\infty$-category $\mathrm{Com}-\operatorname{coalg}(\mathcal{C})$ of commutative coalgebras in $\mathcal{C}$. These two $\infty$-categories are related by a pair of mutually adjoint functors

$$
\text { Lie-alg }(\mathcal{C}) \underset{\text { Prim[-1] }}{\stackrel{\mathrm{C}}{\rightleftarrows}} \operatorname{Com}-\operatorname{coalg}(\mathcal{C}) \text {, }
$$

where the functor $\mathrm{C}$ is the functor computing Lie algebra homology, and the functor Prim is the derived functor of taking primitive elements.

The above functors are not in general equivalences of $\infty$-categories. However, they are for a particular class of tensor $\infty$-categories $\mathcal{C}$ that we call pro-nilpotent, and the $\infty$-category $\mathfrak{D}(\operatorname{Ran} X)$ is such. This will imply our main result:

Theorem 1.2.4. The functors $\mathrm{C}^{\mathrm{ch}}$ and $\operatorname{Prim}^{\mathrm{ch}}[-1]$ in $\mathfrak{D}(\operatorname{Ran} X)$ define an equivalence

$$
\text { Lie- } \operatorname{alg}^{\operatorname{ch}}(\operatorname{Ran} X) \simeq \operatorname{Com}-\operatorname{coalg}^{\mathrm{ch}}(\operatorname{Ran} X)
$$

of $\infty$-categories. Moreover, this equivalence induces an equivalence between the $\infty$-subcategories of chiral Lie algebras and factorization coalgebras on $X$ :

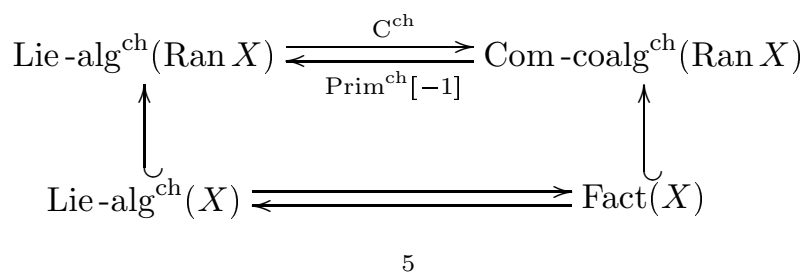


In Section 6 we will apply Theorem 1.2.4 to study chiral Lie algebras obtained by the taking the chiral envelope of a Lie ${ }^{\star}$ algebra. In particular, we will rederive the BD1] computation of chiral homology of such chiral Lie algebras.

In Section 7 we will extend this theorem to include a statement about chiral modules for chiral Lie algebras.

1.2.5. Nilpotence. Let us comment on the pro-nilpotence condition for a tensor $\infty$-category $\mathcal{C}$, and why it implies that the functors (1.2) equivalences in this case.

At least conjecturally, one can modify both sides in (1.2) to turn it into an equivalence. Namely, one has to replace the $\infty$-category $\operatorname{Lie}-\operatorname{alg}(\mathcal{C})$ by its full subcategory Lie-alg ${ }^{\text {nil }}(\mathcal{C})$ consisting of pronilpotent Lie algebras. And one has to replace the $\infty$-category Com-coalg $(\mathcal{C})$ by its full subcategory Com-coalg ${ }^{\text {nil }}(\mathcal{C})$ consisting of ind-nilpotent commutative coalgebras. We refer the reader to Section 3.4 for the precise formulation of this conjecture.

The main feature of the pro-nilpotence condition on $\mathcal{C}$ is that in this case the inclusions

$$
\text { Lie-alg }{ }^{\text {nil }}(\mathcal{C}) \hookrightarrow \operatorname{Lie}-\operatorname{alg}(\mathcal{C}) \text { and } \mathrm{Com}-\operatorname{coalg}^{\text {nil }}(\mathcal{C}) \hookrightarrow \operatorname{Com}-\operatorname{coalg}(\mathcal{C})
$$

are equivalences.

However, unfortunately, in order to actually prove that (1.2) is an equivalence for $\mathcal{C}=\mathfrak{D}(\operatorname{Ran} X)$ we use more than just the above mentioned fact about the inclusions (1.3): Our definition of pronilpotence is quite stringent and explicitly specifies $\mathcal{C}$ as an inverse limit of $\infty$-categories with vanishing $n$-fold tensor products.

\section{3. $\infty$-categories.}

1.3.1. In this work, we study aspects of the homotopy theory of certain algebro-geometric structures. Classically, such as in the study of chain complexes, a notion of a homotopy theory is provided by the homotopy category, a category modulo some equivalence relation. This notion is very useful for a number of purposes, but it is insufficient for many others - for instance, differential graded algebras should have a homotopy theory, but it cannot be extracted with any facility from the homotopy category of complexes. Another, richer, notion of a homotopy theory is provided by Quillen's theory model categories, a category equipped with specified types of morphisms: cofibrations, fibrations, and weak equivalences. Quillen's notion is powerful and sufficient for many purposes, but it, in some sense, has more structure than just the homotopy theory. If we were to allow an analogy with linear algebra, the homotopy/triangulated category is like the rank of a module, and a model category is like a module together with a choice of basis: The homotopy theory itself, like the module, is something in between. Further, working with bases can be very useful in algebra, but they only exist if the module is free, and some constructions are easier coordinate-free; similar is true in homotopy theory.

In the present work, this intermediate notion of homotopy theory will be that of an $\infty$-category. Intuitively, an $\infty$-category consists of the structure of objects, maps, homotopies between maps, homotopies between homotopies, and so forth. Such a structure is provided, for instance, by a category enriched in chain complexes or topological spaces. Topological and DG categories are simple to define, but suffer from technical drawbacks, and we instead use Joyal's quasi-category model for $\infty$-category theory, where this data is just a particular type of simplicial set, satisfying the weak Kan condition of Boardman-Vogt, BB]. This theory has been developed in great detail by Joyal in [Jo] and Lurie in [L1] and [L2, which will be our primary references. The key feature to make note of is that limits, colimits, and functors in the $\infty$-category setting correspond to homotopy limits, homotopy colimits, and derived functors in the setting of DG or model categories. It will be safe to replace the words " $\infty$-category" by "topological category" to obtain the intuitive sense of the results in this work, keeping this one proviso in mind. 
For further motivation for $\infty$-category theory, we refer to Section 2.1 of $[\mathrm{BFN}]$ and, more fundamentally, to the first chapter of [L1].

\subsubsection{Conventions regarding $\infty$-categories. We shall use the following notation:}

By $\infty$-Cat we shall denote the $(\infty, 1)$-category of $\infty$-categories. By $\infty$-Cat ${ }^{\text {st }}$ we shall denote the non-full subcategory of $\infty$-Cat consisting of stable categories and exact functors.

By $\infty$-Cat pres $\subset \infty$-Cat we shall denote the full subcategory consisting of presentable $\infty$-categories. By

$$
\infty \text {-Cat }_{\text {pres }, L} \subset \infty \text {-Cat } \text { pres }
$$

we shall denote the non-full subcategory where we restrict functors to those commuting with colimits. The adjoint functor theorem (Corollary 5.5.2.9 of [L1]) says that a functor between two objects of $\infty$-Cat $_{\text {pres }}$ preserves colimits, i.e., is a 1-morphism in $\infty$-Cat pres,$L_{L}$, if and only if it admits a right adjoint.

We let $\infty$-Cat $t_{\text {pres }}^{\text {st }}$ be the full subcategory of $\infty$ - $\mathrm{Cat}^{\text {st }}$ equal to the preimage of $\infty$-Cat ${ }_{\text {pres }} \subset \infty$-Cat under the forgetful functor $\infty$-Cat ${ }^{\text {st }} \rightarrow \infty$-Cat. We let $\infty$-Cat $t_{\text {pres }, L}^{\text {st }}$ be the non-full subcategory of $\infty$-Cat pres equal to the preimage of $\infty$-Catpres, $L \subset \infty$-Catpres under the above forgetful functor.

We will also use the notation

$$
\infty-\mathrm{Cat}_{\text {pres,cont }}^{\text {st }}:=\infty-\mathrm{Cat}_{\text {pres }, L}^{\mathrm{st}},
$$

and call its 1-morphisms continuous functors. An exact functor between two stable presentable categories is continuous if and only if it commutes with filtered colimits, or, equivalently, with arbitrary direct sums.

Using [L2], Sect. 6.3.1 (and, specifically, Example 6.3.1.22), the category $\infty$-Cat $\mathrm{tres}_{\text {prent }}^{\text {st }}$ is endowed with a symmetric monoidal structure under tensor product.

When discussing a monoidal/symmetric monoidal structure on a stable presentable symmetric monoidal category, unless specified otherwise, we shall mean a structure of associative/commutative algebra object in $\infty$-Cat $t_{\text {pres,cont }}^{\text {st }}$ with respect to the above symmetric monoidal structure on it.

For a ground field $k$, we shall denote by Vect $k$ the commutative algebra object of $\infty$-Cat $t_{\text {pres,cont }}^{\text {st }}$ given by the $\infty$-category associated to the simplicial category of chain complexes of $k$-vector spaces.

Terminology: We use the word "equivalence" in reference to a functor between $\infty$-categories. We will use the term "homotopy equivalence" in reference to a 1-morphism inside a given $\infty$-category (the notion that for an ordinary category would be translated as "isomorphism").

\subsection{D-modules.}

1.4.1. The naive approach. Let $Y$ be a scheme of finite type. Assume for simplicity that $Y$ is separated. We can attach to it a stable $\infty$-category $\mathfrak{D}(Y)$. Namely, we start with the abelian category $\mathfrak{D}(Y)^{\mathcal{Q}}$. When $Y$ is smooth, this is the abelian category of right D-modules over the ring of differential operators on $Y$; for $Y$ singular one defines this category by locally embedding $Y$ into a smooth scheme and using Kasiwara's theorem (see also BD1, Sect. 2.1.3).

To construct $\mathfrak{D}(Y)$, we consider the DG category of complexes over $\mathfrak{D}(Y)^{\mathcal{P}}$, and following $[\mathrm{Dr}$, form the DG quotient by the subcategory of acyclic complexes. It is well-known that to any DG category one can canonically attach a simplicial category, and $\mathfrak{D}(Y)$ is the $\infty$-category associated to this simplicial category. By construction, the category $\mathfrak{D}(Y)$ is cocomplete and compactly generated; in particular, it is presentable.

The question of functoriality $Y \mapsto \mathfrak{D}(Y)$ is less well understood. With some work we can extend the above assignment to a functor

$$
\text { Sch } \longrightarrow \infty-\text { Cat }_{\text {pres,cont }}^{\text {st }},
$$


such that for a map $f: Y_{1} \rightarrow Y_{2}$ the resulting functor $\mathfrak{D}\left(Y_{1}\right) \rightarrow \mathfrak{D}\left(Y_{2}\right)$ at the level of homotopy categories is given by the D-module push-forward, denoted $f_{*}$. We will denote the functor of (1.4) by $\mathfrak{D}^{*}$.

One can also extend the assignment $Y \leadsto \mathfrak{D}(Y)$ differently. Namely, one can construct a functor

$$
\mathrm{Sch}^{\mathrm{op}} \longrightarrow \infty-\mathrm{Cat}_{\text {pres,cont }}^{\mathrm{st}},
$$

such that for a map $f: Y_{1} \rightarrow Y_{2}$ the resulting functor $\mathfrak{D}\left(Y_{2}\right) \rightarrow \mathfrak{D}\left(Y_{1}\right)$ at the level of homotopy categories is given by the D-module pullback, denoted $f^{!}$. We will denote the functor of (1.5) by $\mathfrak{D}^{!}$.

However, for most applications that involve $\infty$-categories, considering the above two functors $\mathfrak{D}^{*}$

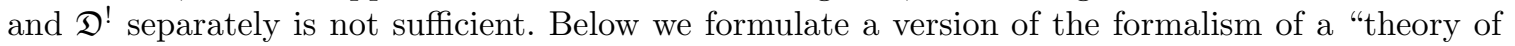
D-modules" which is sufficient for the applications that the authors are aware of.

Remark 1.4.2. To the best of our knowledge, the construction of the theory of D-modules as formulated below does not have a reference in the literature, although many papers on the subject implicitly assume its existence. We hope, however, that this theory will be documented soon 3

1.4.3. The theory of $D$-modules. Let $\mathrm{Sch}^{\text {corr }}$ denote the $(1,1)$-category whose objects are schemes of finite type, and morphisms are correspondences, i.e., for $Y_{1}, Y_{2} \in \operatorname{Sch}^{\text {corr }}$, then $\operatorname{Hom}_{\operatorname{Sch}}{ }^{\text {corr }}\left(Y_{1}, Y_{2}\right)$ is the groupoid of diagrams, an element $f$ in which is of the form

$$
Y_{1} \stackrel{f^{l}}{\longleftarrow} Z \stackrel{f^{r}}{\longrightarrow} Y_{2},
$$

where maps in this groupoid are defined naturally. For a correspondence as in (1.6) we shall symbolically denote by $\left(f^{l}, Z, f^{r}\right)$ the corresponding morphism in $\mathrm{Sch}^{\text {corr }}$.

The composition of morphisms in $\mathrm{Sch}^{\text {corr }}$ is defined naturally by forming Cartesian products. The unit morphism $Y \rightarrow Y$ is one where the maps $f^{l}$ and $f^{r}$ are both isomorphisms. The category $\mathrm{Sch}^{\text {corr }}$ has a natural symmetric monoidal structure given by products.

The category Sch $^{\text {corr }}$ contains a non-full subcategory denoted Sch*, equivalent to the usual category Sch, which has the same objects, but where the morphisms are restricted to have $f^{l}$ an isomorphism. We have another non-full subcategory $\mathrm{Sch}^{!} \subset \mathrm{Sch}^{\text {corr }}$, equivalent to $\mathrm{Sch}^{\mathrm{op}}$, which also has the same objects, but where the morphisms are restricted to have $f^{r}$ an isomorphism.

We assume "the theory of D-modules" in the following format: We assume having a symmetric monoidal functor

$$
\mathfrak{D}^{\bullet}: \mathrm{Sch}^{\text {corr }} \rightarrow \infty-\mathrm{Cat}_{\text {pres,cont }}^{\mathrm{st}},
$$

whose value on a scheme $Y$ is the $\infty$-category $\mathfrak{D}(Y)$, and for a morphism as in (1.6) the functor $\mathfrak{D}\left(Y_{1}\right) \rightarrow \mathfrak{D}\left(Y_{2}\right)$ is given by

$$
f \bullet:=\left(f^{r}\right)_{*} \circ\left(f^{l}\right)^{!} .
$$

Remark 1.4.4. Modulo homotopy theory, the content of the functor (1.7) is the base change theorem: For a Cartesian square in Sch

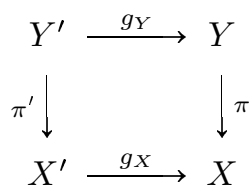

we have a canonical homotopy equivalence $g_{X}^{!} \circ \pi_{*} \simeq \pi_{*}^{\prime} \circ g_{Y}^{!}$.

Restricting the functor $\mathfrak{D}^{\bullet}$ to the subcategories $\mathrm{Sch}^{*}$ and $\mathrm{Sch}^{!}$, we obtain symmetric monoidal functors $\mathfrak{D}^{*}$ and $\mathfrak{D}^{!}$of (1.4) and (1.5), respectively.

\footnotetext{
${ }^{3}$ The corresponding theory in a related context of ind-coherent sheaves has been developed in Ga2].
} 
1.4.5. Let us observe that the theory of D-modules given by (1.7) encodes also the standard adjunctions:

It follows from the definitions that if $g: Y \rightarrow X$ is a locally closed embedding, we have a natural homotopy equivalence in $\operatorname{Hom}_{\mathrm{Sch}^{\mathrm{corr}}}(Y, Y)$

$$
(\mathrm{id}, Y, g) \circ(g, Y, \mathrm{id}) \simeq \operatorname{id}_{Y},
$$

inducing the homotopy equivalence of functors

$$
g^{!} \circ g_{*} \simeq \operatorname{Id}_{\mathfrak{D}(Y)} .
$$

If $g$ is a closed embedding $g=\imath$, then the resulting map $\operatorname{Id}_{\mathfrak{D}(Y)} \rightarrow \imath^{!} \circ \imath_{*}$ is the unit of the $\left(\imath_{*}, \imath^{!}\right)$ adjunction.

If $g$ is an open embedding $g=\jmath$, then the resulting map $\jmath^{!} \circ \jmath_{*} \rightarrow \operatorname{Id}_{\mathfrak{D}(Y)}$ is the counit of the $\left(\jmath^{!}, \jmath_{*}\right)$ adjunction.

Note on notation: To be consistent with the notation from [BD1, for an open embedding $\jmath$, we will often write $\jmath^{*}$ instead of $\jmath$.

Thus, the restriction of $\mathfrak{D}^{*}$ to the non-full subcategory of $\mathrm{Sch}^{\text {open }} \subset \mathrm{Sch}$ with the same objects but open embeddings as morphisms, is a functor

$$
\mathrm{Sch}^{\text {open }} \rightarrow \infty-\text { Cat }_{\text {pres,cont }}^{\text {st }},
$$

obtained from $\left.\left.\mathfrak{D}^{!}\right|_{(\text {Sch }}{ }^{\text {open }}\right)^{\text {op }}$ by taking right adjoints.

1.4.6. Let now $g: Y \rightarrow X$ be an arbitrary separated map, and let $\Delta(Y / X)$ be the diagonal

$$
Y \rightarrow Y \underset{X}{\times} Y
$$

From the $\left(\Delta(Y / X)_{*}, \Delta(Y / X)^{!}\right)$adjunction above, we obtain a canonical map

$$
\operatorname{Id}_{\mathfrak{D}(Y)} \rightarrow g^{!} \circ g_{*} .
$$

When $g$ is proper this map is a unit for the $\left(g_{*}, g^{!}\right)$adjunction.

Thus, the restriction of $\mathfrak{D}_{*}$ to the non-full subcategory of $\mathrm{Sch}^{\text {proper }} \subset \mathrm{Sch}$ with the same objects but proper maps as morphisms, is a functor

$$
\mathrm{Sch}^{\text {proper }} \rightarrow \infty-\text { Cat }_{\text {pres,cont }}^{\text {st }}
$$

obtained from $\left.\left.\mathfrak{D}^{!}\right|_{(\text {Sch }}{ }^{\text {proper }}\right)^{\text {op }}$ by taking left adjoints.

1.4.7. For future use let us note that the functor

$$
\mathfrak{D}^{!}: \mathrm{Sch}^{\mathrm{op}} \rightarrow \infty-\mathrm{Cat}_{\text {pres,cont }}^{\mathrm{st}}
$$

naturally factors through the $(\infty, 1)$-category of commutative algebras in $\infty$-Cat $\mathrm{Cr}_{\text {pres,cont }}^{\text {st }}$. Indeed, this structure is induced by the canonical coalgebra structure on every $Y \in$ Sch given by the diagonal map. 
1.5. Acknowledgements. This work originated in the course of Mike Hopkins' seminar on chiral algebras at Harvard during the fall of 2007, and we thank all the seminar participants for their collaboration and fellowship. We especially thank Jacob Lurie for many conversations, during which many of the present ideas were jointly conceived, and for numerous explanations on various topics addressed in this paper 4 We warmly thank Sasha Beilinson for conversations on chiral algebras and for his generous encouragement on first hearing of this work. DG thanks Nick Rozenblyum for many helpful discussions. JF thanks Reimundo Heluani for first explaining chiral algebras to him as a first-year graduate student, which led him to [BD1] and the comments of 3.3.13 therein. Finally, we wish to thank the anonymous referee, whose remarks have helped to improve the paper.

JF is supported by an NSF postdoctoral fellowship. JF's travel to Cambridge, during which a draft of this paper was written, was supported by the Midwest Topology Network. DG is supported by NSF grant DMS - 0600903 .

\section{Chiral Algebras And FACTORization COAlgEbras}

\subsection{D-modules on the Ran space.}

2.1.1. Let fSet $^{\text {surj }}$ denote the category of non-empty finite sets and surjective morphisms. Let $X^{\mathrm{fSet}^{\text {surj }}}$ denote the functor (fSet ${ }^{\text {surj }}$ ) $^{\text {op }} \rightarrow$ Sch given by $I \leadsto X^{I}$. By composing with the functor

$$
\mathfrak{D}^{!}:(\mathrm{Sch})^{\mathrm{op}} \longrightarrow \infty-\mathrm{Cat}_{\text {pres,cont }}^{\mathrm{st}}
$$

we obtain a functor

$$
\mathfrak{D}^{!}\left(X^{\mathrm{fSet}}{ }^{\text {surj }}\right): \mathrm{fSet}^{\text {surj }} \longrightarrow \infty-\mathrm{Cat}_{\text {pres,cont }}^{\text {st }} .
$$

Definition 2.1.2. The $\infty$-category $\mathfrak{D}(\operatorname{Ran} X)$ is the limit of the functor in (2.1) in $\infty$-Cat $\mathrm{pres}_{\text {, cont }}^{\mathrm{st}}$.

For a finite set $I$, we will denote by $\left(\Delta^{I}\right)^{!}$the tautological functor $\mathfrak{D}(\operatorname{Ran} X) \rightarrow \mathfrak{D}\left(X^{I}\right)$ corresponding to evaluation on $I$. For $I=\mathrm{pt}$, we shall denote $\left(\Delta^{I}\right)^{!}$also by $\left(\Delta^{\text {main }}\right) !$.

2.1.3. Let us recall the following general paradigm. Let $K$ be a small category, and let

$$
\Phi: K \rightarrow \infty-\mathrm{Cat}_{\mathrm{pres}}^{\mathrm{st}}
$$

be a functor. Assume that for every arrow $\alpha: k_{1} \rightarrow k_{2}$ in $K$, the corresponding functor

$$
\Phi_{\alpha}: \Phi_{k_{1}} \rightarrow \Phi_{k_{2}}
$$

admits a left adjoint (which is automatically a 1-morphism in $\infty$-Cat $t_{\text {pres,cont }}^{\text {st }}$ ).

Then we can extend the assignment

$$
i \leadsto \Phi_{i},\left(\alpha: k_{1} \rightarrow k_{2}\right) \leadsto\left(\Phi_{\alpha}\right)^{\mathrm{L}}
$$

to a functor $\Phi^{\mathrm{L}}: K^{\mathrm{op}} \rightarrow \infty-\mathrm{Cat}_{\text {pres,cont }}^{\text {st }}$. Moreover, we have a canonical equivalence (see e.g., GL:DG, Lemma 1.3.3):

$$
\lim _{K} \Phi \simeq \operatorname{colim}_{K^{\mathrm{op}}} \Phi^{\mathrm{L}},
$$

where the colimit is taken in the $(\infty, 1)$-category $\infty$-Cat $t_{\text {pres,cont }}^{\text {st }}$.

Remark 2.1.4. Note that the forgetful functor

$$
\infty-\mathrm{Cat}_{\text {pres,cont }}^{\mathrm{st}} \rightarrow \infty-\mathrm{Cat}^{\mathrm{st}}
$$

commutes with limits, but not with colimits. So, whereas the $\infty$-category in the left-hand side in (2.2) can be calculated in either $\infty-\mathrm{Cat}_{\text {pres,cont }}^{\mathrm{st}}$ or $\infty-\mathrm{Cat}^{\mathrm{st}}$, it is crucial that the right-hand side is calculated in $\infty$-Cat ${ }_{\text {pres,cont }}^{\text {st }}$.

\footnotetext{
${ }^{4}$ Unfortunately, Jacob has declined to sign this work as a coauthor.
} 
2.1.5. Applying (2.2) to $K=\operatorname{fSet}^{\text {surj }}$ and $\Phi=\mathfrak{D}^{!}\left(X^{\mathrm{fSet}}{ }^{\mathrm{surj}}\right)$, we obtain that $\mathfrak{D}(\operatorname{Ran} X)$ can be written as a colimit as follows:

$$
\underset{\left(\mathrm{fSet}^{\mathrm{surj}}\right)^{\mathrm{op}}}{\operatorname{colim}} \mathfrak{D}^{*}\left(X^{\mathrm{fSet}^{\mathrm{surj}}}\right)
$$

Here $\mathfrak{D}^{*}\left(X^{\mathrm{fSet}}{ }^{\text {surj }}\right)$ is the functor $\left(\mathrm{fSet}^{\mathrm{surj}}\right)^{\mathrm{op}} \rightarrow \infty-\mathrm{Cat}_{\text {pres,cont }}^{\text {st }}$ equal to the composition

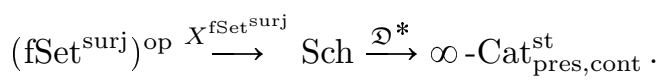

For a finite set $I$, we will denote by $\left(\Delta^{I}\right)_{*}$ the tautological functor $\mathfrak{D}\left(X^{I}\right) \rightarrow \mathfrak{D}(\operatorname{Ran} X)$. By construction, this functor is the left adjoint of $\left(\Delta^{I}\right)^{!}$.

For $I=\mathrm{pt}$, we will denote $\left(\Delta^{I}\right)_{*}$ also by $\left(\Delta^{\text {main }}\right)_{*}$. The following is straightforward:

Lemma 2.1.6. The adjunction $\mathrm{Id} \rightarrow\left(\Delta^{\text {main }}\right)^{!} \circ\left(\Delta^{\text {main }}\right)_{*}$ is a homotopy equivalence.

Corollary 2.1.7. The functor $\left(\Delta^{\text {main }}\right)_{*}: \mathfrak{D}(X) \rightarrow \mathfrak{D}(\operatorname{Ran} X)$ is fully faithful.

2.2. Symmetric monoidal structures on $\mathfrak{D}(\operatorname{Ran} X)$. We shall now recall the definition of the $\star$ and chiral symmetric monoidal structures on $\mathfrak{D}(\operatorname{Ran} X)$, borrowed from [BD1, Sect. 3.4.10.

We shall first give a definition based on the formalism of the theory of D-modules formulated in Section 1.4.1. We shall subsequently write it down more concretely as functors

$$
\mathfrak{D}(\operatorname{Ran} X)^{\otimes J} \rightarrow \mathfrak{D}(\operatorname{Ran} X)
$$

for every finite set $J$.

Both versions of the definition may be difficult to parse. We refer the reader to Section 2.5.4 where this definition is reinterpreted in the context of sheaves on a topological space, which makes it more transparent.

2.2.1. Let us recall the following general paradigm. Let $K$ be a small symmetric monoidal category, and let $\Psi: K \rightarrow \mathcal{A}$ be a right lax symmetric monoidal functor, where $\mathcal{A}$ is another symmetric monoidal category closed under colimits. Then

$$
\underset{K}{\operatorname{colim}} \Psi \in \mathcal{A}
$$

is a commutative algebra object in $\mathcal{A}$.

2.2.2. We shall apply this to $K:=\left(\mathrm{fSet}^{\text {surj }}\right)^{\mathrm{op}}$ and $\mathcal{A}:=\infty$-Cat $_{\text {pres,cont }}^{\text {st }}$, where $\left(\mathrm{fSet}^{\text {surj }}\right)^{\text {op }}$ is viewed as a symmetric monoidal category via the operation of disjoint union.

The functor $\Psi$ will be the composition of $\mathfrak{D} \bullet: \operatorname{Sch}^{\text {corr }} \rightarrow \infty$-Cat $\mathrm{t}_{\text {pres,cont }}^{\text {st }}$, preceded by either of two right lax symmetric monoidal functors:

$$
\left(X^{\mathrm{fSet}}{ }^{\text {surj }}\right)^{\star} \text { and }\left(X^{\mathrm{fSet}^{\text {surj }}}\right)^{\mathrm{ch}}:\left(\mathrm{fSet}^{\text {surj }}\right)^{\mathrm{op}} \rightarrow \mathrm{Sch}^{\mathrm{corr}} .
$$

We let the functor $\left(X^{\mathrm{fSet}}{ }^{\text {surj }}\right)^{\star}$ be the functor

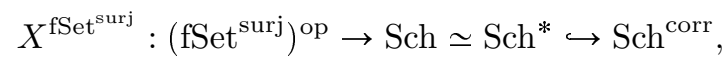

equipped with a natural symmetric monoidal structure. Note that this functor is not only right lax monoidal, but actually monoidal. 
2.2.3. The functor $\left(X^{\mathrm{fSet}}{ }^{\mathrm{surj}}\right)^{\mathrm{ch}}$ is defined as follows. As a functor $\left(\mathrm{fSet}^{\mathrm{surj}}\right)^{\mathrm{op}} \rightarrow \mathrm{Sch}^{\text {corr }}$, it equals $X^{\mathrm{fSet}}{ }^{\text {surj }}$. However, the lax symmetric monoidal structure is different:

Let $I_{J}$ be a collection of finite sets, parameterized by another finite set $J: j \leadsto I_{j}$, which we can also think of as a surjection

$$
\underset{j \in J}{\sqcup} I_{j}=: \stackrel{\pi}{\rightarrow} J
$$

Let $U(\pi)$ be the open subset of $X^{I}$ equal to the locus

$$
\left\{i \leadsto x_{i} \in X, i \in I \mid x_{i_{1}} \neq x_{i_{2}} \text { if } \pi\left(i_{1}\right) \neq \pi\left(i_{2}\right)\right\} \text {, }
$$

and let

$$
\jmath(\pi): U(\pi) \hookrightarrow X^{I}
$$

denote the corresponding open embedding.

We define the right lax symmetric monoidal structure on $\left(X^{\mathrm{fSet}}{ }^{\text {surj }}\right)^{\text {ch }}$ by letting the arrow

$$
\prod_{j \in J} X^{I_{j}} \rightarrow X^{I} \in \mathrm{Sch}^{\text {corr }}
$$

be given by the correspondence

$$
\prod_{j \in J} X^{I_{j}} \stackrel{\jmath(\pi)}{\longleftarrow} U(\pi) \stackrel{\jmath(\pi)}{\longrightarrow} X^{I}
$$

\subsection{Explicit description of tensor product functors.}

2.3.1. Using the presentation of $\mathfrak{D}(\operatorname{Ran} X)$ as a colimit given by (2.3), in order to define a functor as in (2.4), it suffices to define a functor

$$
m_{J}: \underbrace{\left(\mathrm{fSet}^{\mathrm{surj}}\right)^{\mathrm{op}} \times \ldots \times\left(\mathrm{fSet}^{\mathrm{surj}}\right)^{\mathrm{op}}}_{J} \longrightarrow\left(\mathrm{fSet}^{\mathrm{surj}}\right)^{\mathrm{op}}
$$

and a natural transformation between the resulting two functors

$$
\begin{aligned}
& \underbrace{\left(\mathrm{fSet}^{\text {surj }}\right)^{\mathrm{op}} \times \ldots \times\left(\mathrm{fSet}^{\text {surj }}\right)^{\mathrm{op}}}_{J} \rightrightarrows \infty-\mathrm{Cat}_{\text {pres,cont }}^{\text {st }} \text { : } \\
& \left(I_{J} \leadsto \underset{j \in J}{\otimes} \mathfrak{D}\left(X^{I_{j}}\right)\right) \Rightarrow\left(I_{J} \leadsto \mathfrak{D}\left(X^{m_{J}\left(I_{J}\right)}\right)\right),
\end{aligned}
$$

where we denote by $I_{J}$ an object of $\underbrace{\left(\mathrm{fSet}^{\mathrm{surj}}\right)^{\mathrm{op}} \times \ldots \times\left(\mathrm{fSet}^{\mathrm{surj}}\right)^{\mathrm{op}}}_{J}$ as in Section 2.2.3.

For both monoidal structures, we let $m_{J}$ to be the functor of disjoint union:

$$
I_{J} \mapsto I:=\underset{j \in J}{\sqcup} I_{j} .
$$

2.3.2. For the $\star$ symmetric monoidal structure, denoted symbolically $\otimes^{\star}$, we let the natural transformation of (2.5) to be the external tensor product:

$$
\left(M^{I_{j}} \in \mathfrak{D}\left(X^{I_{j}}\right)\right) \leadsto\left(\bigotimes_{j} M^{I_{j}} \in \mathfrak{D}\left(X^{I}\right)\right) .
$$

Note that for objects $M_{j} \in \mathfrak{D}(\operatorname{Ran} X), j \in J$ and a finite set $I$ equipped with a surjection $\pi: I \rightarrow J$, there exists a canonical map

$$
\underset{j \in J}{\bigotimes_{j \in J}}\left(\left(\Delta^{I_{j}}\right)^{!}\left(M_{j}\right)\right) \longrightarrow\left(\Delta^{I}\right)^{!}\left(\underset{j \in J}{\otimes^{\star}} M_{j}\right) .
$$


2.3.3. For the chiral symmetric monoidal structure, denoted symboliccally $\otimes^{\text {ch }}$, we define the natural transformation (2.5) as

$$
\left(M^{I_{j}} \in \mathfrak{D}\left(X^{I_{j}}\right)\right) \leadsto\left(\jmath(\pi)_{*} \circ \jmath(\pi)^{*}\left(\underset{j}{\bigotimes} M^{I_{j}}\right) \in \mathfrak{D}\left(X^{I}\right)\right) .
$$

Note that for objects $M_{j} \in \mathfrak{D}(\operatorname{Ran} X), j \in J$ and a finite set $I$ equipped with a surjection $\pi: I \rightarrow J$, there exists a canonical map

$$
\jmath(\pi)_{*} \circ \jmath(\pi)^{*}\left(\underset{j \in J}{\bigotimes}\left(\Delta^{I_{j}}\right)^{!}\left(M_{j}\right)\right) \longrightarrow\left(\Delta^{I}\right)^{!}\left(\underset{j \in J}{\otimes_{j}^{\mathrm{ch}}} M_{j}\right) .
$$

The following assertion results from the definitions:

Lemma 2.3.4. For $M_{j} \in \mathfrak{D}(\operatorname{Ran} X), j \in J$ and $I$ as above, the resulting map

$$
\underset{\pi}{\oplus} \jmath(\pi)_{*} \circ \jmath(\pi)^{*}\left(\underset{j \in J}{\bigotimes}\left(\Delta^{I_{j}}\right)^{!}\left(M_{j}\right)\right) \longrightarrow\left(\Delta^{I}\right)^{!}\left(\underset{j \in J}{\otimes_{j}^{\mathrm{ch}}} M_{j}\right) .
$$

is a homotopy equivalence, where the direct sum is taken over all surjections $\pi: I \rightarrow J$.

2.4. Chiral Lie algebras and factorization coalgebras. We now define the $\infty$-categories which will be our primary objects of study.

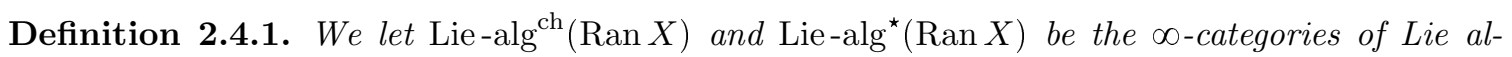
gebras in the $\infty$-category $\mathfrak{D}(\operatorname{Ran} X)$ equipped with the chiral and $\star$ symmetric monoidal structure, respectively.

Definition 2.4.2. The $\infty$-category of chiral Lie and $\star$-Lie algebras on $X$ are the full $\infty$-subcategories

$$
\text { Lie-alg }{ }^{c h}(X) \subset \operatorname{Lie-alg}{ }^{c h}(\operatorname{Ran} X) \text { and Lie-alg* }(X) \subset \operatorname{Lie-alg*}(\operatorname{Ran} X) \text {, }
$$

respectively, spanned by objects for which the underlying $D$-module is supported on $X$, i.e., it lies in the essential image of the functor $\left(\Delta^{\text {main }}\right)_{*}: \mathfrak{D}(X) \rightarrow \mathfrak{D}(\operatorname{Ran} X)$.

Remark 2.4.3. Our names for the above objects are slightly different from those in BD1: What they call a "chiral algebra" we call a "chiral Lie algebra on $X$ "; what they call a "Lie*-algebra" we call a " $\star$-Lie algebra on $X$."

Remark 2.4.4. Throughout this text we will be working with non-unital chiral Lie algebras. The precise relation between non-unital chiral Lie algebras and unital ones will be discussed in another publication. See also Remark 6.4.6.

On the coalgebraic side, we have:

Definition 2.4.5. Com-coalg $^{\mathrm{ch}}(\operatorname{Ran} X)$ is the $\infty$-category of (nonunital) chiral commutative coalgebras for the chiral monoidal structure on $\mathfrak{D}(\operatorname{Ran} X)$.

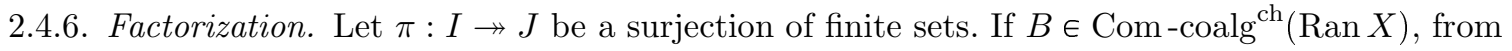
Lemma 2.3.4 we obtain a map

$$
\left(\Delta^{J}\right)^{!}(B) \longrightarrow \jmath(\pi)_{*} \circ \jmath(\pi)^{*}\left(\underset{j \in J}{\bigotimes}\left(\Delta^{I_{j}}\right)^{!}(B)\right)
$$

and by adjunction a map

$$
\jmath(\pi)^{*}\left(\left(\Delta^{J}\right)^{!}(B)\right) \longrightarrow \jmath(\pi)^{*}\left(\underset{j \in J}{\bigotimes}\left(\Delta^{I_{j}}\right)^{!}(B)\right) .
$$

Definition 2.4.7. We say that $B \in \mathrm{Com}-\operatorname{coalg}^{\mathrm{ch}}(\operatorname{Ran} X)$ is a factorization coalgebra if the maps (2.8) are homotopy equivalences for all $I$ and $\pi$. 
We define Com-coalg ${ }_{\text {Fact }}^{\text {ch }}(\operatorname{Ran} X)$ to be the full subcategory of Com-coalg ${ }^{\text {ch }}(\operatorname{Ran} X)$ spanned by factorization coalgebras. We shall also use the notation

$$
\operatorname{Fact}(X):=\text { Com-coalg }{ }_{\text {Fact }}^{\text {ch }}(\operatorname{Ran} X) .
$$

We conclude this subsection with several remarks.

Remark 2.4.8. In [BD1, Sect. 3.4.4, the above category $\operatorname{Fact}(X)$ is denoted $\mathcal{F} \mathcal{A}(X)$, and its objects are referred to as factorization algebras. Our realization of this category as the full subcategory of a certain category of coalgebras rather than algebras is Verdier-biased: The latter would have also been possible if the functors $\jmath(\pi)$ ! had been defined on all of $\mathfrak{D}(U(\pi))$, and not only on the holonomic subcategory. However, putting the definition of [BD1] in the $\infty$-categorical framework, one can give a Verdier self-dual definition of $\operatorname{Fact}(X)$ by requiring a homotopy-coherent system of homotopy equivalences (2.8). For that reason it seems most preferable to term objects of Fact $(X)$ as "factorization D-modules."

Remark 2.4.9. The $\infty$-categories Lie-alg ${ }^{\operatorname{ch}}(\operatorname{Ran} X)$ and $\mathfrak{D}(X)$ are both presentable $\infty$-categories, and they both can be made equivalent to the simplicial nerve of model categories. Their intersection, the $\infty$-category of chiral algebras Lie-alg ${ }^{\text {ch }}(X)$, is however not presentable: It fails, for instance, to have coproducts. As a consequence, the $\infty$-category of chiral Lie algebras does not arise as the simplicial nerve of a model category. The same holds true on the coalgebra side and for Fact $(X)$.

Remark 2.4.10. A chiral commutative coalgebra may be thought of as a lax factorization D-module, i.e., a D-module for which there are given the factorizing structure maps (as in (2.8)), but which are no longer necessarily homotopy equivalences. The factorization property is closely related to locality in quantum field theory, so one might think of general chiral commutative coalgebras as related to field theories in which the condition of locality is weakened. General chiral commutative coalgebras are thus unlikely to be especially physically compelling, but it is still convenient to allow for this mathematical generalization.

\subsection{Variant: the topological context.}

2.5.1. In this subsection we let $X$ be a Hausdorff locally compact topological space. We consider the functor

$$
I \leadsto X^{I}
$$

from (fSet $\left.{ }^{\text {surj }}\right)^{\text {op }}$ to the category Top cl. $_{\text {l.c. }}$ of Hausdorff locally compact topological spaces and maps that are closed embeddings.

Consider the functor

$$
\mathrm{Shv}^{!}:\left(\mathrm{Top}_{\mathrm{cl}}^{\text {l.c. }}\right)^{\mathrm{op}} \longrightarrow \infty-\mathrm{Cat}_{\text {pres }}^{\mathrm{st}}
$$

that assigns to $\mathcal{Y} \in \operatorname{Top}_{\mathrm{cl}}^{\text {l.c. }}$ the $\infty$-category $\operatorname{Shv}(\mathcal{Y})$, and for a closed embedding $f: \mathcal{Y}_{1} \rightarrow \mathcal{Y}_{2}$ the corresponding functor $f^{!}$. (Here $\operatorname{Shv}(-)$ stands for $\infty$-category of sheaves of $k$-vector spaces, where $k$ is a field of characteristic 0 .)

Composing, we obtain the functor

$$
\operatorname{Shv}^{!}\left(X^{\mathrm{fSet}}{ }^{\text {surj }}\right): \mathrm{fSet}^{\text {surj }} \longrightarrow \infty-\mathrm{Cat}_{\text {pres }}^{\mathrm{st}}
$$

and we set

$$
\operatorname{Shv}(\operatorname{Ran} X):=\lim _{\mathrm{fSet}^{\mathrm{surj}}} \operatorname{Shv}^{!}\left(X^{\mathrm{fSet}}{ }^{\mathrm{surj}}\right) .
$$

The constructions of Sections 2.2 and 2.4 go through in the present context. In particular, we obtain two symmetric monoidal structures on $\operatorname{Shv}(\operatorname{Ran} X)$, and the notions of chiral Lie algebra,

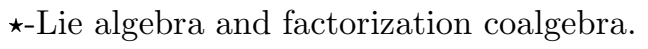

The analog of Theorem 1.2.4 goes through for $\operatorname{Shv}(\operatorname{Ran} X)$ with no modification. 
2.5.2. Let $\mathcal{R}(X)$ be the topological space defined as in BD1, Sect. 3.4.1. We have pair of adjoint functors

$$
\operatorname{Shv}(\operatorname{Ran} X) \rightleftarrows \operatorname{Shv}(\mathcal{R}(X)) .
$$

According to loc.cit., Sect. 4.2.4 we have:

Lemma 2.5.3. The functor $\operatorname{Shv}(\operatorname{Ran} X) \rightarrow \operatorname{Shv}(\mathcal{R}(X))$ is fully faithful.

2.5.4. Let us interpret the $\star$ and chiral symmetric monoidal structures on $\operatorname{Shv}(\operatorname{Ran} X)$ in terms of Lemma 2.5.3

Following BD1, Sect. 3.4.1(iii), the topological space $\mathcal{R}(X)$ is a commutative semigroup with respect to the operation denoted "union" (which corresponds to the operation of taking the union of finite subsets of $X$ ).

The $\star$-monoidal structure is induced by the above semigroup structure on $\mathcal{R}(X)$ by means of the functor of direct image:

$$
\text { union }_{*}: \operatorname{Shv}(\mathcal{R}(X))^{\otimes I} \longrightarrow \operatorname{Shv}(\mathcal{R}(X)) \text {. }
$$

To describe the chiral symmetric monoidal structure, we note that for a finite set $I$, the product $\mathcal{R}(X)^{I}$ contains an open subset

$$
\left(\mathcal{R}(X)^{I}\right)_{\operatorname{disj}} \stackrel{J^{I}}{\rightarrow} \mathcal{R}(X)^{I},
$$

corresponding to $I$-tuples of finite subsets of $X$ are are pairwise disjoint.

The chiral symmetric monoidal structures is given by the functor

$$
\left(i \rightsquigarrow \mathcal{F}_{i} \in \operatorname{Shv}(\mathcal{R}(X)), i \in I\right) \leadsto \operatorname{union}_{*}\left(\left(J^{I}\right)_{*} \circ\left(J^{I}\right)^{*}\left(\underset{i}{\mathbb{F}_{i}} \mathcal{F}_{i}\right)\right) \in \operatorname{Shv}(\mathcal{R}(X)) .
$$

It follows easily from the definitions that the adjoint functors in Lemma 2.5 .3 intertwine the corresponding symmetric monoidal structures on $\operatorname{Shv}(\operatorname{Ran} X)$ and $\operatorname{Shv}(\mathcal{R}(X))$.

2.5.5. Finally, let us remark how the notion of factorization coalgebra in $\operatorname{Shv}(\operatorname{Ran} X)$ relates to that of $\mathcal{E}_{n}$-algebra:

Let us take $X=\mathbb{R}^{n}$. As was communicated to us by Lurie, one has the following assertion:

Theorem 2.5.6. The $\infty$-category of translation-equivariant factorization coalgebras in $\operatorname{Shv}\left(\operatorname{Ran} \mathbb{R}^{n}\right)$ is equivalent to that of $\mathcal{E}_{n}$-coalgebras over $k$.

Remark 2.5.7. This theorem does not formally follow from Theorem 5.3.4.10 of [L2]: One can show that for $X$ being a manifold, the category $\operatorname{Shv}^{!}(\operatorname{Ran} X)$ is equivalent to the category of cosheaves on $\mathcal{R}(X)$ in the colimit topology rather than the topology in which the theorem in L2] is proved. However, according to Lurie, the above result holds for the colimit topology as well.

Remark 2.5.8. Based on the previous remark, we can view the theory of chiral Lie algebras studied in this paper as an algebro-geometric analogue of the theory of $\mathcal{E}_{n}$-algebras. Recall now that on the category of $\mathcal{E}_{n}$-algebras there is a contravariant $\mathcal{E}_{n}$-Koszul duality functor, introduced in GJ].

We should emphasize that chiral Koszul duality studied in this paper is totally unrelated to the $\mathcal{E}_{n}$-Koszul duality, either technically or conceptually.

However, we should add that the $\mathcal{E}_{n}$-Koszul duality does have an interpretation in the factorization setting as a form of Verdier duality of (co)sheaves on the Ran space, as is discussed in F2. In the latter incarnation, $\mathcal{E}_{n}$-Koszul duality has an analogue in the algebro-geometric context of chiral Lie algebras/factorization D-modules, which we hope to discuss in another publication. 


\section{Algebras and coalgebras over (Co) Operads: Recollections}

This section is included for the reader's convenience. None of the results stated here are original. The general reference for operads and algebras over them in the $\infty$-category framework is [L2, Chapters 2 and 3.

\subsection{Operads.}

3.1.1. Let $\mathcal{X}$ be a stable presentable symmetric monoidal $\infty$-category. Let $\mathcal{X}^{\Sigma}$ denote the $\infty$ category of symmetric sequences in $\mathcal{X}$. I.e., objects of $\mathcal{X}^{\Sigma}$ are collections $\mathcal{O}=\{\mathcal{O}(n), n \geqslant 1\}$, where each $\mathcal{O}(n)$ is an object of $\mathcal{X}$ acted on by the symmetric group $\Sigma_{n}$.

The $\infty$-category $\mathcal{X}^{\Sigma}$ has a natural monoidal structure. A convenient way to think about this monoidal structure is the following:

We have a natural functor $\mathcal{X}^{\Sigma} \rightarrow \operatorname{Funct}(\mathcal{X}, \mathcal{X})$ :

$$
(\mathcal{O}=\{\mathcal{O}(n)\}) \leadsto\left(x \leadsto \underset{n \geqslant 1}{\sqcup}\left(\mathcal{O}(n) \otimes x^{\otimes n}\right)_{\Sigma_{n}}\right) .
$$

The monoidal structure on $\mathcal{X}^{\Sigma}$ is designed so that the functor in (3.1) is monoidal.

Definition 3.1.2. The $\infty$-category $\operatorname{Op}(\mathcal{X})$ (resp., $\operatorname{coOp}(\mathcal{X})$ ) of augmented operads (resp., cooperads) in $\mathcal{X}$ is that of augmented associative algebras (resp., coalgebras) in $\mathcal{X}^{\Sigma}$ with respect to the above monoidal structure.

3.1.3. We have a pair of adjoint functors

$$
\operatorname{Bar}: \mathrm{Op}(\mathcal{X}) \rightleftarrows \operatorname{coOp}(\mathcal{X}): \text { Cobar }
$$

see [G], GK, C1].

In fact, the above pair of adjoint functors is a particular case of the adjunction between augmented associative algebras and augmented associative coalgebras, i.e., of one reviewed in Section 3.3 for $\mathcal{O}$ being the associative operad, when we take our ambient monoidal category to be $\mathcal{X}^{\Sigma}$ :

In the case of the associative operad, the ambient category needs to be just monoidal, not symmetric monoidal, and neither does it need to be stable. We only need the monoidal operation to distribute over sifted colimits in each variable 5

Definition 3.1.4. An operad $\mathcal{O} \in \mathrm{Op}(\mathcal{X})$ is derived Koszul if the adjunction map

$$
\mathcal{O} \longrightarrow \operatorname{Cobar} \circ \operatorname{Bar}(\mathcal{O})
$$

is a homotopy equivalence.

Remark 3.1.5. Any Koszul operad in chain complexes in $\mathcal{X}=$ Vect $_{k}$, in the original sense of Ginzburg-Kapranov [GK], is derived Koszul in the above sense.

In fact, any augmented operad for which $1_{\mathcal{X}} \rightarrow \mathcal{O}(1)$ is a homotopy equivalence is derived Koszul. In particular, the Lie operad is derived Koszul (and this is true even for the Lie operad in spectra, see, e.g., [C1]).

\subsection{Algebras over an operad.}

\footnotetext{
${ }^{5}$ We recall that an index category $I$ is called sifted if the diagonal functor $I \rightarrow I \times I$ is homotopy cofinal, see [L1, Definition 5.5.8.1. Filtered categories and $\Delta^{\mathrm{op}}$, the opposite of the simplicial indexing category, are the essential examples.
} 
3.2.1. Let $\mathcal{X}$ be as above. Let $\mathcal{C}$ be a (not necessarily unital) stable presentable symmetric monoidal $\infty$-category compatibly tensored over $\mathcal{X}$, i.e., $\mathcal{C}$ is a commutative algebra object in the $(\infty, 1)$-category of $\mathcal{X}$-modules in $\infty$-Cat pres,cont $^{\text {st }}$.

Formula (3.1) (applied now to $x$ being an object of $\mathcal{C}$ rather than $\mathcal{X}$ ) defines an action of the monoidal category $\mathcal{X}^{\Sigma}$ acts on $\mathcal{C}$. Hence, an operad $\mathcal{O}$ (resp., cooperad $\mathcal{P}$ ) in $\mathcal{X}$ defines a monad $\mathrm{T}_{\mathcal{O}}: \mathcal{C} \rightarrow \mathcal{C}$ (resp., a comonad $\left.\mathrm{S}_{\mathcal{P}}: \mathcal{C} \rightarrow \mathcal{C}\right)$.

Definition 3.2.2. For an operad $\mathcal{O} \in \operatorname{Op}(\mathcal{X})$, the $\infty$-category $\mathcal{O}$-alg $(\mathcal{C})$ of (non-unital) $\mathcal{O}$-algebras in $\mathcal{C}$ is the $\infty$-category of $\mathrm{T}_{\mathcal{O}}$-modules in $\mathcal{C}$.

Remark 3.2.3. The preceding definition of $\mathcal{O}-\operatorname{alg}(\mathcal{C})$ is equivalent to that given by Lurie in the case where $\mathcal{X}$ is the $\infty$-category of topological spaces. We adopt the above definition in order to accommodate the definition of Lie algebras in a symmetric monoidal $\infty$-category, since Lie is an operad in $k$-modules, but not in spaces.

Definition 3.2.4. For a cooperad $\mathcal{P} \in \operatorname{coOp}(\mathcal{X})$, the $\infty$-category $\mathcal{P}$-coalg dil.p. $(\mathcal{C})$ of ind-nilpotent $\mathcal{P}$-coalgebras in $\mathcal{C}$ is the $\infty$-category of $\mathrm{S}_{\mathcal{P}}$-comodules in $\mathcal{C}$.

Remark 3.2.5. We shall introduce the category of "all" (i.e., not necessarily ind-nilpotent) $\mathcal{P}$ coalgebras in Section 3.5. In loc.cit. it will also become clear why we use the terminology "indnilpotent" for $\mathcal{P}$-coalgebras in $\mathcal{C}$.

The subscript "d.p." in $\mathcal{P}$-coalg nil.p. $(\mathcal{C})$ stands for "divided powers." Again, we refer the reader to Section 3.5 where the reason for this notation will become clear.

3.2.6. Let $\mathcal{O}$ be an object of $\mathrm{Op}(\mathcal{X})$. Let oblv $\mathrm{O}_{\mathcal{O}}$ denote the tautological forgetful functor

$$
\mathcal{O}-\operatorname{alg}(\mathcal{C}) \rightarrow \mathcal{C} \text {. }
$$

The functor oblv $v_{\mathcal{O}}$ commutes with limits, and with sifted colimits 6 Let

$$
\text { Free }_{\mathcal{O}}: \mathcal{C} \rightarrow \mathcal{O}-\operatorname{alg}(\mathcal{C})
$$

denote its left adjoint.

In addition, the augmentation on $\mathcal{O}$ defines the functor

$$
\operatorname{triv}_{\mathcal{O}}: \mathcal{C} \rightarrow \mathcal{O}-\operatorname{alg}(\mathcal{C})
$$

The functor triv $\mathcal{O}$ commutes with both limits and colimits.

3.2.7. Let $\mathcal{P}$ be an object of $\operatorname{coOp}(\mathcal{X})$. Let oblv $\mathcal{P}$ denote the forgetful functor

$$
\mathcal{P} \text {-coalg } \text { d.p. }_{\text {nil }}(\mathcal{C}) \rightarrow \mathcal{C} \text {. }
$$

The functor oblv $\mathcal{P}$ commutes with colimits. We let

$$
\operatorname{coFree}_{\mathcal{P}}: \mathcal{C} \rightarrow \mathcal{P} \text {-coalg nil.p. }(\mathcal{C})
$$

denote its right adjoint.

In addition, the augmentation on $\mathcal{P}$ defines the functor

$$
\operatorname{triv}_{\mathcal{P}}: \mathcal{C} \rightarrow \mathcal{P} \text {-coalg d.p. }(\mathcal{C}) .
$$

The functor triv $\mathcal{P}$ commutes colimits.

If the cooperad $\mathcal{P}$ has the property that for every $n$, the functor $c \leadsto \mathcal{P}(n) \otimes c$ distributes over limits, then the functor $\operatorname{triv}_{\mathcal{P}}$ commutes with sifted limits.

The above condition on $\mathcal{P}$ is satisfied in many cases of interest: e.g., if $\mathcal{X}=\operatorname{Vect}_{k}$ and all $\mathcal{P}(n)$ are (bounded complexes of) finite-dimensional vector spaces.

\subsection{Koszul duality functors.}

\footnotetext{
${ }^{6}$ The siftedness condition is used as follows: For a monoidal category $\mathcal{C}$ in which tensor products distribute over colimits, the functor of $n$-th tensor power $c \leadsto c^{\otimes n}$ distributes over sifted colimits.
} 
3.3.1. For $\mathcal{O} \in \operatorname{Op}(\mathcal{X})$, we now consider the left adjoint of the functor triv $\mathcal{O}$, which we denote

$$
\operatorname{Bar}_{\mathcal{O}}: \mathcal{O}-\operatorname{alg}(\mathcal{C}) \rightarrow \mathcal{C}
$$

Remark 3.3.2. At the classical level, since the multiplication on $\operatorname{triv}_{\mathcal{O}}(M)$ is trivial, any map $A \rightarrow$ $\operatorname{triv}_{\mathcal{O}}(M)$ must send to zero any element $a \in A$, which is decomposable, i.e., a multiple of two or more elements (e.g., $a=f \cdot a^{\prime} \cdot a^{\prime \prime}$, for $a^{\prime}, a^{\prime \prime}$ in $A$ and $f$ in $\mathcal{O}(2)$ ). Consequently, the left adjoint assigns to an $\mathcal{O}$-algebra $A$ the indecomposables of $A$, the quotient of $A$ by the decomposable elements. In the instance of classical commutative algebra, this quotient is isomorphic to the cotangent space of the associated pointed affine scheme, so one can geometrically imagine the indecomposables as forming an operadic version of the cotangent space. Returning to the homotopy theory, the left adjoint of $\operatorname{triv}_{\mathcal{O}}$ can be formed in the model category setting as a derived functor of indecomposables, where one resolves an $\mathcal{O}$-algebra and takes indecomposables in the resolution.

Remark 3.3.3. The reason for notation $\operatorname{Bar}_{\mathcal{O}}$ is the following. Let $\mathcal{A}$ be a monoidal $\infty$-category and $\mathcal{M}$ a module category. Then, under some mild hypothesis on $\mathcal{A}$ and $\mathcal{M}$, for associative algebras $R, R^{\prime} \in A$ and a homomorphism $R \rightarrow R^{\prime}$, the functor left adjoint to the forgetful functor $\operatorname{Mod}_{R^{\prime}}(\mathcal{M}) \rightarrow \operatorname{Mod}_{R}(\mathcal{M})$ exists, and is computed as the geometric realization of simplicial object $\operatorname{Bar}\left(R^{\prime}, R,-\right)$., called the bar-construction, i.e.,

$$
\operatorname{Bar}\left(R^{\prime}, R,-\right):=\left|\operatorname{Bar}\left(R^{\prime}, R,-\right) \cdot\right|
$$

(see [L2 or [F1 for a more extended explanation in the context of $\infty$-categories). Here we take $\mathcal{A}=\mathcal{X}^{\Sigma}, \mathcal{M}=\mathcal{C}, R=\mathcal{O}$ and $R^{\prime}=1$.

The definition of the Koszul dual cooperad as the associative coalgebra in $\mathcal{X}^{\Sigma}$ Koszul dual to $\mathcal{O}$ yields:

Lemma 3.3.4. There is a natural homotopy equivalence of comonads acting on $\mathcal{C}$ :

$$
\operatorname{Bar}_{\mathcal{O}} \circ \operatorname{triv}_{\mathcal{O}} \simeq \mathrm{S}_{\mathcal{O}^{v}}
$$

where $\mathcal{O}^{\vee}:=\operatorname{Bar}(\mathcal{O})$

The general theory of monads 7 implies:

Corollary 3.3.5. The functor $\operatorname{Bar}_{\mathcal{O}}: \mathcal{O}-\operatorname{alg}(\mathcal{C}) \rightarrow \mathcal{C}$ factors as

$$
\mathcal{O}-\operatorname{alg}(\mathcal{C}) \stackrel{\operatorname{Bar}_{\mathcal{O}}^{\text {enh }}}{\longrightarrow} \mathcal{O}^{\vee}-\text { coalg d.p. }_{\text {dil }}^{\text {nil }}(\mathcal{C}) \stackrel{\text { oblv }_{\mathcal{O}} \vee}{\longrightarrow} \mathcal{C}
$$

for a canonically defined functor $\operatorname{Bar}_{\mathcal{O}}^{\mathrm{enh}}: \mathcal{O}-\operatorname{alg}(\mathcal{C}) \rightarrow \mathcal{O}^{\vee}-\operatorname{coalg}_{\text {d.p. }}^{\text {nil }}(\mathcal{C})$.

Since the functor $\operatorname{Bar}_{\mathcal{O}}$, being a left adjoint, commutes with colimits, and since oblv $\mathcal{O}_{\mathcal{V}}$ commutes with colimits and is conservative, we obtain that the functor $\operatorname{Bar}_{\mathcal{O}}^{\text {enh }}$ also commutes with colimits.

3.3.6. We can depict the resulting commutative diagrams of functors as follows:

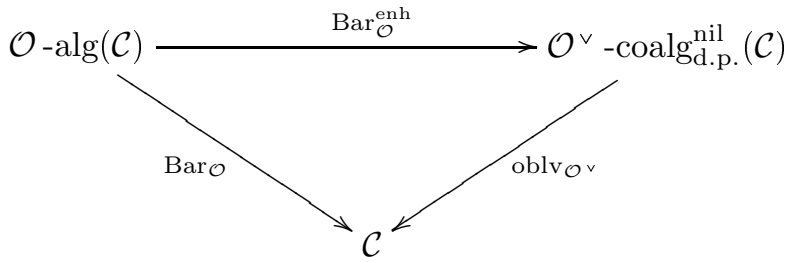

\footnotetext{
${ }^{7}$ Some of this theory is summarized in Section 6.2 and the relevant fact for the next corollary specifically in Section 6.2 .2
} 
and

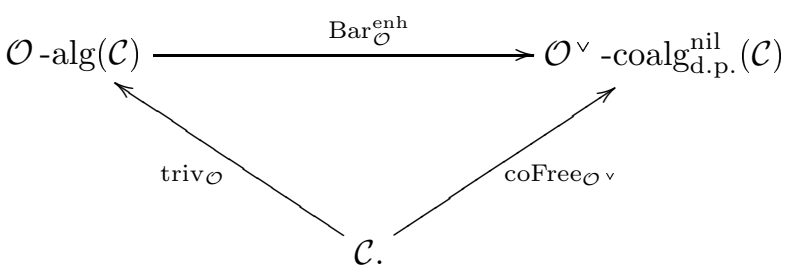

Remark 3.3.7. The relative ease of construction for the above diagram is one the great virtues of $\infty$-category theory. In the setting of model categories, one in general loses the strict monad structure on an adjunction when one passes to derived functors: For example, there is a coherence problem to solve in constructing a coalgebra structure on, say, the bar construction $k \otimes_{A} k$ of an augmented algebra at the chain level, [Mo].

We have also another commutative diagram, namely:

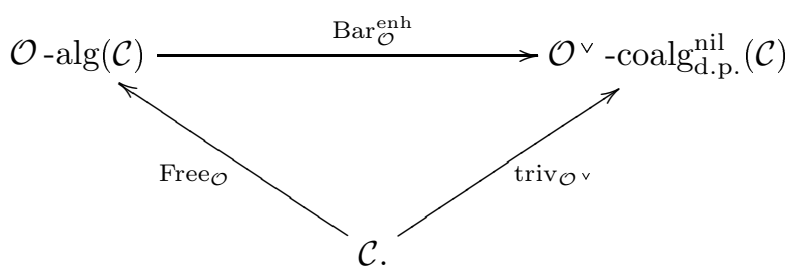

3.3.8. Let $\mathcal{P}$ be an object of $\operatorname{coOp}(\mathcal{X})$, and consider the right adjoint of the functor triv ${ }_{\mathcal{P}}$ :

$$
\operatorname{Cobar}_{\mathcal{P}}: \mathcal{P} \text {-coalg } \text { d.p. }_{\text {il }}(\mathcal{C}) \rightarrow \mathcal{C} \text {. }
$$

As in Lemma 3.3.4 we have:

Lemma 3.3.9. There is a canonical homomorphism of monads

$$
\mathrm{T}_{\mathcal{P} \vee} \rightarrow \operatorname{Cobar}_{\mathcal{P}} \circ \operatorname{triv}_{\mathcal{P}}
$$

where $\mathcal{P}^{\vee}:=\operatorname{Cobar}(\mathcal{P})$.

Remark 3.3.10. Unlike Lemma 3.3.4 the map in the above lemma is no longer a homotopy equivalence, since the action of $\mathcal{X}^{\Sigma}$ on $\mathcal{C}$ does not commute with totalizations.

Corollary 3.3.11. The functor $\operatorname{Cobar}_{\mathcal{P}}: \mathcal{P}$-coalg d.p. $(\mathcal{C}) \rightarrow \mathcal{C}$ factors as

$$
\mathcal{P} \text {-coalg } \text { d.p. }_{\text {il }}(\mathcal{C}) \stackrel{\text { Cobarr }_{\mathcal{P}}^{\text {enh }}}{\longrightarrow} \mathcal{P}^{\vee}-\operatorname{alg}(\mathcal{C}) \stackrel{\text { oblv }_{\mathcal{P}}}{\longrightarrow} \mathcal{C}
$$

for a canonically defined functor $\operatorname{Cobar}_{\mathcal{P}}^{\text {enh }}: \mathcal{P}$-coalg $\cos _{\text {d.p. }}^{\text {nil }}(\mathcal{C}) \rightarrow \mathcal{P}^{\vee}-\operatorname{alg}(\mathcal{C})$.

We can depict the resulting commutative diagram of functors as follows:

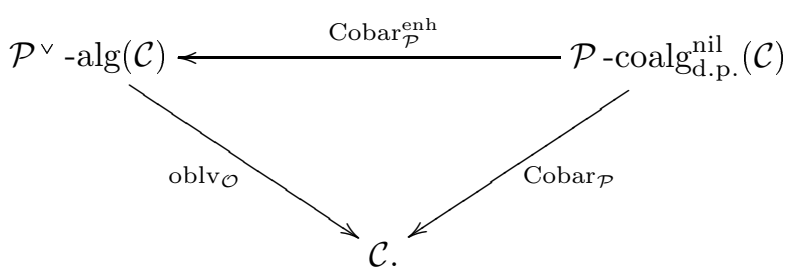


We have also another commutative diagram, namely:

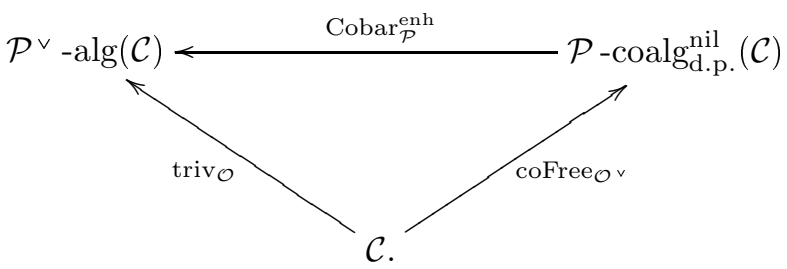

3.3.12. Combining Lemmas 3.3.4 and 3.3.9, we obtain:

For $\mathcal{O}$ and $\mathcal{P}$ as above, let us be given a map $\mathcal{O}^{\vee} \rightarrow \mathcal{P}$, or equivalently, a map $\mathcal{O} \rightarrow \mathcal{P}^{\vee}$. These maps define functors

$$
\mathcal{P}^{\vee}-\operatorname{alg}(\mathcal{C}) \rightarrow \mathcal{O}-\operatorname{alg}(\mathcal{C}) \text { and } \mathcal{O}^{\vee}-\operatorname{coalg}_{\text {d.p. }}^{\text {nil }}(\mathcal{C}) \rightarrow \mathcal{P} \text {-coalg nil.p. }(\mathcal{C}) .
$$

Corollary 3.3.13. The composed functors

$$
\mathcal{O}-\operatorname{alg}(\mathcal{C}) \stackrel{\operatorname{Bar}_{\mathcal{O}}^{\text {enh }}}{\longrightarrow} \mathcal{O}^{\vee}-\operatorname{coalg}_{\text {d.p. }}^{\text {nil }}(\mathcal{C}) \rightarrow \mathcal{P} \text {-coalg } \text { d.p. }_{\text {nil }}(\mathcal{C})
$$

and

are naturally mutually adjoint.

$$
\mathcal{O}-\operatorname{alg}(\mathcal{C}) \leftarrow \operatorname{Cobar}(\mathcal{P})-\operatorname{alg}(\mathcal{C}) \stackrel{\operatorname{Cobar}_{\mathcal{P}}^{\text {enh }}}{\longleftarrow} \mathcal{P} \text {-coalg } \text { d.p. }_{\text {nil }}(\mathcal{C})
$$

\subsection{Turning Koszul duality into an equivalence.}

3.4.1. Suppose that the operad $\mathcal{O}$ is derived Koszul. From the above discussion obtain a pair of adjoint functors:

$$
\operatorname{Bar}_{\mathcal{O}}^{\text {enh }}: \mathcal{O}-\operatorname{alg}(\mathcal{C}) \rightleftarrows \mathcal{O}^{\vee}-\operatorname{coalg}_{\text {d.p. }}^{\text {nil }}(\mathcal{C}): \text { Cobar }_{\mathcal{O} \vee}^{\text {enh }} .
$$

The above adjunction is in general not an equivalence. We shall now describe a procedure how to modify the left-hand side to (conjecturally) turn it into an equivalence.

3.4.2. Let us call an $\mathcal{O}$-algebra $A$ nilpotent, if there exists an integer $n$, such that the maps

$$
\mathcal{O}\left(n^{\prime}\right) \otimes A^{\otimes n^{\prime}} \rightarrow A
$$

are null-homotopic for $n^{\prime} \geqslant n$.

Definition 3.4.3. An $\mathcal{O}$-algebra $A$ is pro-nilpotent if it is equivalent to a limit of nilpotent $A$ algebras.

Let $\mathcal{O}-\operatorname{alg}{ }^{\text {nil }}(\mathcal{C}) \subset \mathcal{O}-\operatorname{alg}(\mathcal{C})$ denote the full subcategory spanned by pro-nilpotent algebras.

It is easy to see that the above embedding admits a left adjoint, which we denote Compl, making $\mathcal{O}-\operatorname{alg}^{\text {nil }}(\mathcal{C})$ a localization of $\mathcal{O}-\operatorname{alg}(\mathcal{C})$.

3.4.4. It follows from the construction that the essential image of the functor

$$
\mathcal{O}-\operatorname{alg}(\mathcal{C}) \leftarrow \mathcal{O}^{\vee} \text {-coalg nil.p. }(\mathcal{C}): \text { Cobar }_{\mathcal{O}^{\vee}}^{\text {enh }}
$$

belongs to $\mathcal{O}$-alg ${ }^{\text {nil }}$. Let us denote the resulting functor

$$
\mathcal{O}-\operatorname{alg}^{\text {nil }}(\mathcal{C}) \leftarrow \mathcal{O}^{\vee} \text {-coalg } \text { d.p. }_{\text {nil }}(\mathcal{C})
$$

by $\mathrm{KD}_{\mathcal{O} \leftarrow \mathcal{O}^{v}}$.

By adjunction, we obtain that the functor $\operatorname{Bar}_{\mathcal{O}}^{\mathrm{enh}}$ factors as

$$
\mathcal{O}-\operatorname{alg}(\mathcal{C}) \stackrel{\text { Compl }}{\longrightarrow} \mathcal{O}-\operatorname{alg}^{\text {nil }}(\mathcal{C}) \longrightarrow \mathcal{O}^{\vee}-\operatorname{coalg}_{\text {d.p. }}^{\text {nil }}(\mathcal{C}),
$$

for a canonically defined functor

$$
\operatorname{KD}_{\mathcal{O} \rightarrow \mathcal{O}^{\vee}}: \mathcal{O}-\operatorname{alg}^{\text {nil }}(\mathcal{C}) \longrightarrow \mathcal{O}^{\vee}-\operatorname{coalg}_{\text {d.p. }}^{\text {nil }}(\mathcal{C})
$$


which is left adjoint to $\mathrm{KD}_{\mathcal{O} \leftarrow \mathcal{O}^{v}}$.

Conjecture 3.4.5. The adjoint functors

$$
\mathrm{KD}_{\mathcal{O} \rightarrow \mathcal{O} \vee}: \mathcal{O}-\operatorname{alg}^{\text {nil }}(\mathcal{C}) \rightleftarrows \mathcal{O}^{\vee}-\operatorname{coalg}_{\text {d.p. }}^{\text {nil }}(\mathcal{C}): \mathrm{KD}_{\mathcal{O} \leftarrow \mathcal{O}} \vee
$$

are equivalences of $\infty$-categories.

In the next section we will give a proof of this conjecture in a particular case.

Remark 3.4.6. The derived notion of Koszul duality discussed here is broadly construed; there is no use made of Koszul resolutions in the sense of [Pr. It would be equally accurate to call this bar-cobar duality.

\subsection{Coalgebras over an operad.}

3.5.1. Note that the monoidal $\infty$-category $\mathcal{X}^{\Sigma}$ of symmetric sequences is endowed with a different right lax action on $\mathcal{C}$, i.e., a lax monoidal functor $\mathcal{X}^{\Sigma} \rightarrow \operatorname{Funct}(\mathcal{C}, \mathcal{C})$ :

$$
(\mathcal{O}=\{\mathcal{O}(n)\}) \leadsto\left(c \rightsquigarrow \prod_{n \geqslant 1}\left(\mathcal{O}(n) \otimes c^{\otimes n}\right)_{\Sigma_{n}}\right) .
$$

Hence, for a cooperad $\mathcal{P} \in \operatorname{coOp}(\mathcal{X})$, it makes sense to talk about $\mathcal{P}$-comodules in $\mathcal{C}$ with respect to this new action. We denote the resulting $\infty$-category of comodules by $\mathcal{P}$-coalg $\cos _{\text {d.p. }}(\mathcal{C})$ and call them $\mathcal{P}$-coalgebras (with divided powers). See $[\mathrm{Fr}$ for a treatment of simplicial $\mathcal{O}$-algebras with divided powers, where it shown, for instance, that a simplicial Lie algebra with divided powers is a simplicial restricted Lie algebra.

Remark 3.5.2. Since the above is only a right lax action, a cooperad $\mathcal{P}$ does not define a comonad in $\mathcal{C}$. In particular, the forgetful functor $\mathcal{P}$-coalg d.p. $(\mathcal{C}) \rightarrow \mathcal{C}$ does not in general admit a right adjoint.

We have an evident forgetful functor

$$
\mathcal{P} \text {-coalg } \text { d.p. }_{\text {nil }}(\mathcal{C}) \longrightarrow \mathcal{P} \text {-coalg } \text { d.p. }_{\text {. }}(\mathcal{C}) \text {. }
$$

Remark 3.5.3. One can show that the above functor $\mathcal{P}$-coalg nil.p. $(\mathcal{C}) \rightarrow \mathcal{P}$-coalg d.p. $_{\text {. }}(\mathcal{C})$ is fully faithful and that it admits right adjoint, making the $\infty$-category $\mathcal{P}$-coalg dil.p. $(\mathcal{C})$ into a colocalization of $\mathcal{P}$ - coalg d.p. $_{\text {d }}(\mathcal{C})$

3.5.4. Note now that we have yet another action (resp., right lax action) of $\mathcal{X}^{\Sigma}$ on $\mathcal{C}$ :

$$
(\mathcal{O}=\{\mathcal{O}(n)\}) \leadsto\left(c \rightsquigarrow \underset{n \geqslant 1}{\sqcup}\left(\mathcal{O}(n) \otimes c^{\otimes n}\right)^{\Sigma_{n}}\right),
$$

and

$$
(\mathcal{O}=\{\mathcal{O}(n)\}) \leadsto\left(c \rightsquigarrow \prod_{n \geqslant 1}\left(\mathcal{O}(n) \otimes c^{\otimes n}\right)^{\Sigma_{n}}\right)
$$

respectively.

Thus, for a cooperad $\mathcal{P} \in \operatorname{coOp}(\mathcal{X})$ we have two more notions of $\mathcal{P}$-coalgebras in $\mathcal{C}$. We denote the corresponding $\infty$-categories by

$$
\mathcal{P} \text {-coalg }{ }^{\text {nil }}(\mathcal{C}) \text { and } \mathcal{P} \text {-coalg }(\mathcal{C}),
$$

respectively. As in the case of divided powers, we have natural forgetful functor

$$
\mathcal{P}-\operatorname{coalg}^{\text {nil }}(\mathcal{C}) \underset{21}{\longrightarrow} \mathcal{P} \text {-coalg }(\mathcal{C})
$$


3.5.5. We also have natural homomorphisms of right lax actions

$$
\text { original action } \rightarrow \text { (3.11) and (3.9) } \rightarrow \text { (3.12) }
$$

given by the trace map

$$
(-)_{\Sigma_{n}} \rightarrow(-)^{\Sigma_{n}}
$$

(i.e., averaging over the group $\Sigma_{n}$ ), and the corresponding functors between the $\infty$-categories of comodules:

$$
\mathcal{P} \text {-coalg d.p. }(\mathcal{C}) \rightarrow \mathcal{P} \text {-coalg }{ }^{\text {nil }}(\mathcal{C}) \text { and } \mathcal{P} \text {-coalg }{ }_{\text {d.p. }}(\mathcal{C}) \rightarrow \mathcal{P} \text {-coalg }(\mathcal{C})
$$

Let us note that when $\mathcal{X}$ is compatibly tensored over Vect $_{k}$, where $k$ has characteristic zero, the above homomorphisms of actions are homotopy equivalences, and hence the functors in (3.14) are equivalences.

\section{Koszul DuALity in NilPotent TENSOR $\infty$-CATEGORIES}

4.1. Nilpotent and pro-nilpotent tensor $\infty$-categories. We retain the setting of Section 3.2 .

Definition 4.1.1. We shall say that $\mathcal{C}$ is pro-nilpotent if it can be exhibited as a limit

$$
\mathcal{C} \simeq \lim _{\mathbb{N o p}} \mathcal{C}_{i}
$$

(where the limit is taken in the $(\infty, 1)$-category of stable symmetric monoidal $\infty$-categories compatibly tensored over $\mathcal{X})$, such that

- $\mathcal{C}_{0}=0$;

- For every $i \geqslant j$, the transition functor $f_{i, j}: \mathcal{C}_{i} \rightarrow \mathcal{C}_{j}$ commutes with limits 8

- For every $i$, the restriction of the tensor product functor $\mathcal{C}_{i} \otimes \mathcal{C}_{i} \rightarrow \mathcal{C}_{i}$ to $\operatorname{ker}\left(f_{i, i-1}\right) \otimes \mathcal{C}_{i}$ is null-homotopic.

We shall say that $\mathcal{C}$ is nilpotent of order $n$, if the functors $f_{i, j}$ are equivalences for $i, j \geqslant n$.

We are going to show:

Proposition 4.1.2. Assume that the operad $\mathcal{O}$ is such that augmentation map $\mathcal{O}(1) \rightarrow 1_{\mathcal{X}}$ is a homotopy equivalence. Assume also that $\mathcal{C}$ is pro-nilpotent. Then the mutually adjoint functors of (3.8) are homotopy equivalences of $\infty$-categories.

Remark 4.1.3. The assumption that the map $\mathcal{O}(1) \rightarrow 1 \mathcal{X}$ is a homotopy equivalence can be weakened. All we actually need is that $\mathcal{O}$ be derived Koszul and that the kernel of $\mathcal{O}(1) \rightarrow 1_{\mathcal{X}}$ act nilpotently on $\mathcal{C}$.

The rest of this subsection is devoted to the proof of the above proposition.

4.1.4. Reduction to the nilpotent case. Let $\mathcal{C}$ be written as $\lim _{\alpha} \mathcal{C}_{\alpha}$, where the transition functors commute with limits and colimits. For each index $\alpha$, let $f_{\alpha}$ denote the evaluation functor $\mathcal{C} \rightarrow \mathcal{C}_{\alpha}$.

The fact that the functors $f_{\alpha, \beta}: \mathcal{C}_{\beta} \rightarrow \mathcal{C}_{\alpha}$ commute with limits (resp., colimits) implies that for every $\alpha$, the functor $f_{\alpha}$ commutes with limits (resp., colimits). I.e., limits (resp., colimits) in $\mathcal{C}$ can be computed "component-wise."

We have

$$
\mathcal{O}-\operatorname{alg}(\mathcal{C}) \simeq \lim _{\alpha} \mathcal{O}-\operatorname{alg}\left(\mathcal{C}_{\alpha}\right)
$$

and this equivalence commutes with the corresponding functors oblv $v_{\mathcal{O}}$ (this requires no assumption on the transition functors). We also have

$$
\mathcal{O}^{\vee}-\operatorname{coalg}_{\text {d.p. }}^{\text {nil }}(\mathcal{C}) \simeq \lim _{\alpha} \mathcal{O}^{\vee}-\operatorname{coalg}_{\text {d.p. }}^{\text {nil }}\left(\mathcal{C}_{\alpha}\right)
$$

\footnotetext{
${ }^{8}$ It is are also required to commute with colimits, according to our conventions, see Section 1.3 .1
} 
and this equivalence commutes with the corresponding functors oblv $\mathrm{O}_{\mathcal{O}}$ (this follows from the above mentioned fact that the functors $f_{\alpha}$ commute with colimits).

Moreover, we claim that for each $\alpha$, the diagram

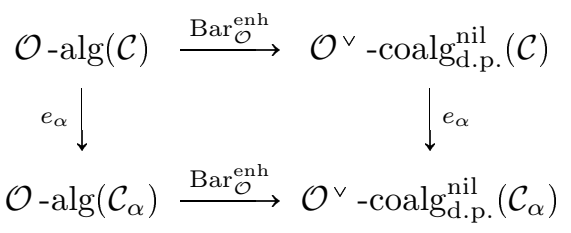

commutes. This again follows from the fact that the functors $f_{\alpha}$ commute with colimits.

The diagram

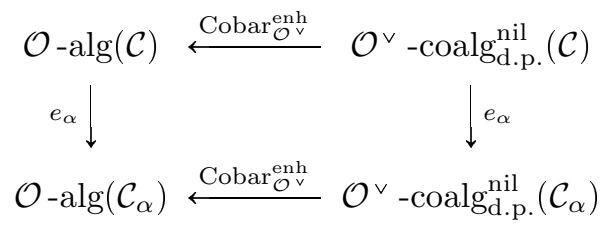

commutes as well, and this follows from the fact that the functors $f_{\alpha}$ commute with limits.

The commutativity of the above two diagrams shows that it if the adjoint functors of (3.8) are equivalences for each $\mathcal{C}_{\alpha}$, then they are also equivalences for $\mathcal{C}$.

4.1.5. The nilpotence condition. Thus, from now on we shall assume that $\mathcal{C}$ is nilpotent. We will use it in the following form:

Lemma 4.1.6. Assume that $\mathcal{C}$ is nilpotent. For any cooperad $\mathcal{P}$ we have:

(a) The functor $\operatorname{Cobar}_{\mathcal{P}}: \mathcal{P}$-coalg nil.p. $(\mathcal{C}) \rightarrow \mathcal{C}$ commutes with sifted colimits.

(b) The map of monads of Lemma 3.3 .9 is a homotopy equivalence.

Proof. We prove point (a):

By construction, the functor $\operatorname{Cobar}_{\mathcal{P}}$ is the composition of a functor

$$
\operatorname{Cobar}_{\mathcal{P}}^{\bullet}: \mathcal{P} \text {-coalg }{ }_{\text {d.p. }}^{\text {nil }}(\mathcal{C}) \rightarrow \mathcal{C}^{\Delta}
$$

which commutes with sifted colimits (because the $n$-fold tensor power functor commutes with sifted colimits), followed by the functor

$$
\text { Tot }: \mathcal{C}^{\Delta} \rightarrow \mathcal{C}
$$

Here we denote by $\mathcal{C}^{\Delta}=\operatorname{Funct}(\Delta, \mathcal{C})$ the $\infty$-category of cosimplicial objects in $\mathcal{C}$, and Tot is the functor of taking the limit over $\Delta$.

Let $\mathcal{C}$ be such that all $n$-fold tensor products are equivalent to zero. This implies that for $A \in$ $\mathcal{P}$-coalg dil.p. $_{\text {nil }}(\mathcal{C})$, the natural map

$$
\operatorname{Cobar}_{\mathcal{P}}^{\bullet}(A) \rightarrow \operatorname{cosk}^{\leqslant n}\left(\left.\operatorname{Cobar}_{\mathcal{P}}^{\bullet}(A)\right|_{\Delta_{\leqslant n}}\right)
$$

is a homotopy equivalence, where $\Delta_{\leqslant n} \subset \Delta$ is the subcategory spanned by objects of cardinality $\leqslant n$. Hence,

$$
\left.\operatorname{Cobar}_{\mathcal{P}}(A) \simeq \lim _{\Delta_{\leqslant n}} \operatorname{Cobar}_{\mathcal{P}}^{\bullet}(A)\right|_{\Delta_{\leqslant n}}
$$

As was mentioned above, the functor

$$
\left.A \leadsto \operatorname{Cobar}_{\mathcal{P}}^{\bullet}(A)\right|_{\Delta_{\leqslant n}}: \mathcal{P} \text {-coalg } \cos _{\text {d.p. }}^{\text {nil }}(\mathcal{C}) \longrightarrow \mathcal{C}^{\Delta_{\leqslant n}}
$$

commutes with sifted colimits. Hence, the assertion follows from the fact that the functor of limit over $\Delta_{\leqslant n}$

$$
\mathcal{C}^{\Delta \leqslant n} \rightarrow \mathcal{C}
$$

commutes with colimits (since $\mathcal{C}$ is stable and $\Delta_{\leqslant n}$ is finite, the limit diagram is equivalent to a colimit one). 
To prove point (b), let $\operatorname{Cobar}^{\bullet}(\mathcal{P})$ be the canonical cosimplicial object of $\mathcal{X}^{\Sigma}$, such that

$$
\operatorname{Tot}\left(\operatorname{Cobar}^{\bullet}(\mathcal{P})\right) \simeq \operatorname{Cobar}(\mathcal{P})=: \mathcal{P}^{\vee} .
$$

Then $\mathrm{T}_{\mathcal{P}} \vee$ is given by

and $\operatorname{Cobar}_{\mathcal{P}} \circ \operatorname{triv}_{\mathcal{P}}$ is given by

$$
c \leadsto \operatorname{Tot}\left(\operatorname{Cobar}^{\bullet}(\mathcal{P})\right) \cdot(c)
$$$$
\left.c \leadsto \operatorname{Tot} \operatorname{Cobar}^{\bullet}(\mathcal{P}) \cdot(c)\right),
$$

where - - denotes the action of $\mathcal{X}^{\Sigma}$ on $\mathcal{C}$.

However, as above, the maps

and

$$
\operatorname{Tot}\left(\operatorname{Cobar}^{\bullet}(\mathcal{P})\right) \cdot(c) \rightarrow\left(\left.\lim _{\Delta_{\leqslant n}} \operatorname{Cobar}^{\bullet}(\mathcal{P})\right|_{\Delta_{\leqslant n}}\right) \cdot c
$$

$$
\operatorname{Tot}\left(\operatorname{Cobar}^{\bullet}(\mathcal{P}) \cdot(c)\right) \rightarrow \lim _{\Delta_{\leqslant n}}\left(\left.\operatorname{Cobar}^{\bullet}(\mathcal{P}) \cdot(c)\right|_{\Delta_{\leqslant n}}\right)
$$

are homotopy equivalences. Thus, the above totalizations are isomorphic to finite limits, and since $\mathcal{C}$ is stable, also to colimits. Therefore, the assertion follows from the fact that the action of $\mathcal{X}$ on $\mathcal{C}$ and the monoidal operation on $\mathcal{C}$ commute with colimits.

Since the functor oblv $\mathcal{P}_{\vee}$ is conservative and commutes with colimits, from point (a) of Lemma 4.1.6 we obtain:

Corollary 4.1.7. The functor $\operatorname{Cobar}_{\mathcal{P}}^{\mathrm{enh}}: \mathcal{P}$-coalg nil $(\mathcal{C}) \rightarrow \mathcal{P}^{\vee}$-alg $(\mathcal{C})$ commutes with geometric realizations.

4.1.8. The functor $\operatorname{Bar}_{\mathcal{O}}^{\text {enh }}$ is fully faithful. To prove that $\operatorname{Bar}_{\mathcal{O}}^{\text {enh }}$ is fully faithful we need to show that the unit of the adjunction

$$
\mathrm{Id} \longrightarrow \operatorname{Cobar}_{\mathcal{O}}^{\mathrm{enh}} \circ \operatorname{Bar}_{\mathcal{O}}^{\mathrm{enh}}
$$

is a homotopy equivalence.

Since every object of $\mathcal{O}-\operatorname{alg}(\mathcal{C})$ can be obtained as a geometric realization of a simplicial object whose terms lie in the essential image of the functor

$$
\text { Free }_{\mathcal{O}}: \mathcal{C} \longrightarrow \mathcal{O}-\operatorname{alg}(\mathcal{C})
$$

from Corollary 4.1.7 we obtain that it is enough to show that the map

$$
\text { Free }_{\mathcal{O}} \longrightarrow \operatorname{Cobar}_{\mathcal{O}}^{\mathrm{enh}} \circ \operatorname{Bar}_{\mathcal{O}}^{\mathrm{enh}} \circ \operatorname{Free}_{\mathcal{O}}
$$

is a homotopy equivalence. Again, since the forgetful functor oblv $\mathcal{O}: \mathcal{O}-\operatorname{alg}(\mathcal{C}) \rightarrow \mathcal{C}$ is conservative, it is enough to show that the induced map

$$
\operatorname{oblv}_{\mathcal{O}} \circ \text { Free }_{\mathcal{O}} \longrightarrow \operatorname{oblv}_{\mathcal{O}} \circ \operatorname{Cobar}_{\mathcal{O}^{\vee}}^{\mathrm{enh}} \circ \operatorname{Bar}_{\mathcal{O}}^{\mathrm{enh}} \circ \mathrm{Free}_{\mathcal{O}}
$$

is a homotopy equivalence.

By definition, the functor in the left-hand side of (4.3) identifies with $\mathrm{T}_{\mathcal{O}}$. We can rewrite the right-hand side of (4.3) as

$$
\operatorname{Cobar}_{\mathcal{O}} \vee \circ \operatorname{Bar}_{\mathcal{O}}^{\mathrm{enh}} \circ \text { Free }_{\mathcal{O}}
$$

From Diagram (3.5), we obtain a canonical homotopy equivalence of functors

$$
\operatorname{Bar}_{\mathcal{O}}^{\text {enh }} \circ \text { Free }_{\mathcal{O}} \simeq \operatorname{triv}_{\mathcal{O}} \vee
$$

Hence, the map in (4.3) can be thought of as a map

$$
\mathrm{T}_{\mathcal{O}} \longrightarrow \operatorname{Cobar}_{\mathcal{O}} \text { } \text { o } \operatorname{triv}_{\mathcal{O}} \vee
$$

However, it is easy to see from the construction that the map in (4.4) equals the composition

$$
\left.\mathrm{T}_{\mathcal{O}} \longrightarrow \mathrm{T}_{(\mathcal{O} \vee}\right)^{\vee} \longrightarrow \text { Cobar }_{\mathcal{O} \vee} \operatorname{otriv}_{\mathcal{O}} \vee
$$


where the first arrow is given by (4.3), and the second arrow is given by Lemma 3.3.9. Hence, the fact that $\mathcal{O}$ is derived Koszul (see Remark 3.1.5) and Lemma 4.1.6)(b) imply that the map in (4.4) is a homotopy equivalence.

4.1.9. Proof of the equivalence. To prove that the functor $\operatorname{Bar}_{\mathcal{O}}^{\mathrm{enh}}$ is an equivalence, it remains to show that its right adjoint, namely $\operatorname{Cobar}_{\mathcal{O}^{v}}^{\mathrm{enh}}$, is conservative. For that it is sufficient to show that the functor

$$
\operatorname{Cobar}_{\mathcal{P}}: \mathcal{P} \text {-coalg } \operatorname{dil}_{\text {d.p. }}^{\text {nil }}(\mathcal{C}) \longrightarrow \mathcal{C}
$$

is conservative for a cooperad $\mathcal{P}$, provided that $\mathcal{C}$ is nilpotent.

Remark 4.1.10. Note that Conjecture 3.4.5 would imply that the functor Cobar $\mathrm{P}_{\mathcal{P}}^{\mathrm{nh}}$ is conservative for any $\mathcal{C}$, without the nilpotence (or pro-nilpotence) assumption.

Let $\mathcal{C}_{i}$ be as in Definition 4.1.1. Let $f_{i}: \mathcal{C} \rightarrow \mathcal{C}_{i}$ denote the corresponding evaluation functors.

Let $\alpha: B_{1} \rightarrow B_{2}$ be a map in $\mathcal{P}$-coalg $\ln _{\text {d.p. }}^{\text {nil }}(\mathcal{C})$ that is not a homotopy equivalence. Let $i$ be the minimal integer such that the map

$$
f_{i}(\alpha): f_{i}\left(B_{1}\right) \rightarrow f_{i}\left(B_{2}\right)
$$

is not a homotopy equivalence.

For any $B \in \mathcal{P}$-coalg d.p. $(\mathcal{C})$ we have a canonical map

$$
\operatorname{Cobar}_{\mathcal{P}}(B)=\lim _{\Delta} \operatorname{Cobar}_{\mathcal{P}}^{\bullet}(B) \longrightarrow \operatorname{oblv}_{\mathcal{P}}(B)
$$

By the choice of the index $i$, the map

$$
f_{i} \circ \operatorname{Cobar}_{\mathcal{P}}(\alpha): f_{i} \circ \operatorname{Cobar}_{\mathcal{P}}\left(B_{1}\right) \longrightarrow f_{i} \circ \operatorname{Cobar}_{\mathcal{P}}\left(B_{2}\right),
$$

induces a homotopy equivalence

$$
\operatorname{coker}\left(\operatorname{Cobar}_{\mathcal{P}}\left(B_{1}\right) \longrightarrow B_{1}\right) \longrightarrow \operatorname{coker}\left(\operatorname{Cobar}_{\mathcal{P}}\left(B_{2}\right) \longrightarrow B_{2}\right) \text {. }
$$

Hence, the map (4.6) is not a homotopy equivalence. Hence $\operatorname{Cobar}_{\mathcal{P}}(\alpha)$ is not a homotopy equivalence, as required.

4.2. Coalgebras vs. ind-nilpotent coalgebras in the pro-nilpotent case. In the following, we retain the assumption that $\mathcal{C}$ is pro-nilpotent:

Proposition 4.2.1. The functors (3.10) and (3.13) are equivalences.

Proof. As in the proof of Proposition 4.1 .2 we immediately reduce to the case when $\mathcal{C}$ is nilpotent. In the latter case, in both cases (with or without divided powers), the two right lax actions of $\mathcal{X}^{\Sigma}$ on $\mathcal{C}$ are tautologically equivalent by the nilpotence condition.

\subsection{The case of Lie algebras.}

4.3.1. Let $\mathcal{X}$ be the category Vect $_{k}$, where $k$ has characteristic zero. We shall consider the augmented operad Lie, obtained from the usual (non-unital) Lie operad by formally adjoining the unit. As was mentioned in Remark 3.1.5, the operad Lie is derived Koszul. 
4.3.2. Let $\mathcal{C}$ be a (not necessarily unital) stable symmetric monoidal $\infty$-category, compatibly tensored over $\operatorname{Vect}_{k}$.

Let Lie-alg $(\mathcal{C})$ denote the $\infty$-category of Lie algebras in $\mathcal{C}$, and let Com-coalg $(\mathcal{C})$ denote the category of non-unital commutative coalgebras on $\mathcal{C}$. Recall that we have a pair of adjoint functors:

$$
\mathrm{C}: \operatorname{Lie}-\operatorname{alg}(\mathcal{C}) \rightleftarrows \text { Com-coalg }(\mathcal{C}): \operatorname{Prim}[-1],
$$

where $\mathrm{C}$ is the functor of the homological Chevalley complex, and Prim is the (derived) functor of primitive elements (here [-1] stands for the cohomological shift by 1 to the right, i.e., the loop functor).

We claim that we have proved the following:

Proposition 4.3.3. Assume that $\mathcal{C}$ is pro-nilpotent. Then the functors in (4.7) are equivalences of $\infty$-categories.

Proof. It is known (see [GK], C1]) that the cooperad Lie ${ }^{\vee}$ identifies with $\operatorname{Com}[1]$, i.e., $\operatorname{Com}[1](n)=$ $k[n-1]$ with the sign action of $\Sigma_{n}$ for every $n$. Moreover, the functor

$$
\operatorname{Bar}_{\text {Lie }}^{\text {enh }}: \operatorname{Lie}-\operatorname{alg}(\mathcal{C}) \longrightarrow \operatorname{Com}[1]-\operatorname{coalg}_{\text {d.p. }}^{\text {nil }}(\mathcal{C}) \longrightarrow \operatorname{Com}[1]-\operatorname{coalg}_{\text {d.p. }}(\mathcal{C}) \longrightarrow \operatorname{Com}[1]-\operatorname{coalg}(\mathcal{C})
$$

is the functor $\mathrm{C}[-1]$.

From Proposition 4.1 .2 we obtain an equivalence of $\infty$-categories

$$
\operatorname{Bar}_{\text {Lie }}^{\text {enh }}: \operatorname{Lie}-\operatorname{alg}(\mathcal{C}) \longrightarrow \operatorname{Com}[1]-\text { coalg d.p. }_{\text {dil }}^{\text {nil }}(\mathcal{C}) .
$$

By Proposition 4.2.1 the pro-nilpotence assumption on $\mathcal{C}$ implies that the functor

$$
\operatorname{Com}[1]-\operatorname{coalg}^{\text {nil }}(\mathcal{C}) \longrightarrow \operatorname{Com}[1]-\operatorname{coalg}(\mathcal{C})
$$

is an equivalence.

Due to the characteristic zero assumption, the functor

$$
\mathrm{Com}[1]-\operatorname{coalg}_{\text {d.p. }}^{\text {nil }}(\mathcal{C}) \longrightarrow \operatorname{Com}[1]-\operatorname{coalg}^{\text {nil }}(\mathcal{C})
$$

is an equivalence as well.

Thus, we obtain that $\mathrm{C} \simeq \operatorname{Bar}_{\mathrm{Lie}}^{\mathrm{enh}}[-1]$ defines an equivalence

$$
\text { Lie-alg }(\mathcal{C}) \longrightarrow \text { Com-coalg }(\mathcal{C}) .
$$

\section{Proof of the MAIN THEOREM}

5.1. Koszul duality in the chiral setting. Our current goal is to prove the first part of Theorem 1.2 .4

Theorem 5.1.1. The above functors

$$
\mathrm{C}^{\text {ch }}: \text { Lie-alg }{ }^{\text {ch }}(\operatorname{Ran} X) \rightleftarrows \text { Com-coalg }{ }^{\text {ch }}(\operatorname{Ran} X): \operatorname{Prim}^{\text {ch }}[-1]
$$

are mutually inverse equivalences of $\infty$-categories.

In view of Proposition 4.3.3, it suffices to show that the $\infty$-category $\mathfrak{D}(\operatorname{Ran} X)$, equipped with the chiral symmetric monoidal structure, is pro-nilpotent. 
5.1.2. Let $n$ be a positive integer. For a finite set $I$, let $X^{I, \leqslant n}$ be the closed subscheme of $X^{I}$ equal to the union of the images of the diagonal maps $\Delta(\pi): X^{J} \rightarrow X^{I}$ for all surjections $\pi: I \rightarrow J$ with $|J| \leqslant n$. Let $X^{I,>n} \subset X^{I}$ be the complementary open subset. Let

$$
\imath^{I, n}: X^{I, \leqslant n} \hookrightarrow X^{I} \hookleftarrow X^{I,>n}: g^{I, n}
$$

denote the corresponding maps.

We obtain the functors

$$
X^{\Delta, \leqslant n}, X^{\Delta,>n}:\left(\mathrm{fSet}^{\mathrm{surj}}\right)^{\mathrm{op}} \longrightarrow \mathrm{Sch}
$$

and the corresponding functors

$$
\mathfrak{D}^{!}\left(X^{\Delta, \leqslant n}\right), \mathfrak{D}^{!}\left(X^{\Delta,>n}\right): \mathrm{fSet}^{\mathrm{surj}} \longrightarrow \infty-\mathrm{Cat}^{\mathrm{st}} .
$$

Let $\mathfrak{D}\left(\operatorname{Ran}^{\leqslant n} X\right)$ and $\mathfrak{D}\left(\operatorname{Ran}^{>n} X\right)$ denote the corresponding $\infty$-categories

$$
\lim _{\text {fSet }^{\text {surj }}} \mathfrak{D}^{!}\left(X^{\Delta, \leqslant n}\right) \text { and } \lim _{\text {fSet }^{\text {surj }}} \mathfrak{D}^{!}\left(X^{\Delta,>n}\right),
$$

respectively.

5.1.3. For a surjection $\pi: I_{1} \rightarrow I_{2}$, the map $\Delta(\pi): X^{I_{1}} \rightarrow X^{I_{2}}$ sends

$$
X^{I_{1}, \leqslant n} \longrightarrow X^{I_{2}, \leqslant n} \text { and } X^{I_{1},>n} \longrightarrow X^{I_{2},>n} .
$$

Hence, we obtain commutative diagrams of functors

$$
\begin{aligned}
& \mathfrak{D}\left(X^{I_{1}, \leqslant n}\right) \stackrel{\left(\imath^{I_{1}, n}\right)^{!}}{\longleftarrow} \mathfrak{D}\left(X^{I_{1}, n}\right) \stackrel{\left(J^{I_{1}, n}\right)^{*}}{\longrightarrow} \mathfrak{D}\left(X^{I_{1},>n}\right) \\
& \Delta(\pi)^{!} \downarrow \quad \Delta(\pi)^{!} \downarrow \quad \downarrow \Delta(\pi)^{!} \\
& \mathfrak{D}\left(X^{I_{2}, \leqslant n}\right) \stackrel{\left(\imath^{I_{2}, n}\right)^{!}}{\longleftarrow} \mathfrak{D}\left(X^{I_{2}, n}\right) \stackrel{\left(J^{I_{2}, n}\right)^{*}}{\longrightarrow} \mathfrak{D}\left(X^{I_{2},>n}\right)
\end{aligned}
$$

and their adjoints:

$$
\begin{aligned}
& \mathfrak{D}\left(X^{I_{1}, \leqslant n}\right) \stackrel{\left(\imath^{I_{1}, n}\right) *}{\longrightarrow} \mathfrak{D}\left(X^{I_{1}, n}\right) \stackrel{\left(J^{I_{1}, n}\right) *}{\longleftarrow} \mathfrak{D}\left(X^{I_{1},>n}\right) \\
& \Delta(\pi)^{!} \downarrow \quad \Delta(\pi)^{!} \downarrow \quad \downarrow \Delta(\pi)^{!} \\
& \mathfrak{D}\left(X^{I_{2}, \leqslant n}\right) \stackrel{\left(\imath^{I_{2}, n}\right) *}{\longrightarrow} \mathfrak{D}\left(X^{I_{2}, n}\right) \stackrel{\left(J^{I_{2}, n}\right) *}{\longleftarrow} \mathfrak{D}\left(X^{I_{2},>n}\right)
\end{aligned}
$$

So, we obtain adjoint pairs of functors

$$
\left(\imath^{n}\right)_{*}: \mathfrak{D}\left(\operatorname{Ran}^{\leqslant n} X\right) \rightleftarrows \mathfrak{D}(\operatorname{Ran} X):\left(\imath^{n}\right)^{!} \text {and }\left(\jmath^{n}\right)^{*}: \mathfrak{D}(\operatorname{Ran} X) \rightleftarrows \mathfrak{D}\left(\operatorname{Ran}^{>n} X\right):\left(\jmath_{n}\right)_{*}
$$

that commute with the evaluation maps $\left(\Delta^{I}\right)^{!}$. Moreover, the functors $\left(\imath^{n}\right)_{*}$ and $\left(\jmath_{n}\right)_{*}$ are fully faithful, and

$$
\mathfrak{D}\left(\operatorname{Ran}^{\leqslant n} X\right) \rightleftarrows \mathfrak{D}(\operatorname{Ran} X) \rightleftarrows \mathfrak{D}\left(\operatorname{Ran}^{>n} X\right)
$$

is a short exact sequence of stable $\infty$-categories: I.e., the category on the right is the localization of the category in the middle with respect to the category on the left.

Similarly, we have the corresponding maps and functors for any pair $n_{1} \leqslant n_{2}$.

Lemma 5.1.4. The functor

$$
\left\{\left(\imath^{n}\right)^{!}\right\}: \mathfrak{D}(\operatorname{Ran} X) \longrightarrow \lim _{n} \mathfrak{D}\left(\operatorname{Ran}^{\leqslant n} X\right)
$$

is an equivalence.

Proof. This follows from the fact that each $\left(\imath^{I, n}\right)^{!}$is an equivalence as soon as $n \geqslant|I|$. 
5.1.5. From Lemma 2.3.4 we obtain that for any $n$, the essential image of $\mathfrak{D}\left(\operatorname{Ran}^{>n} X\right)$ under $\left(\jmath_{n}\right)_{*}$ is a monoidal ideal with respect to the chiral symmetric monoidal structure on $\mathfrak{D}(\operatorname{Ran} X)$, i.e., the product of any object with an object in the essential image of $\mathfrak{D}\left(\operatorname{Ran}^{>n} X\right)$ remains in the essential image of $\mathfrak{D}\left(\operatorname{Ran}^{>n} X\right) \cdot 9$ As a consequence, the localization of $\mathfrak{D}(\operatorname{Ran} X)$ with respect to $\mathfrak{D}\left(\operatorname{Ran}^{>n} X\right)$ obtains a monoidal structure, such that the localization functor is a homomorphism of monoidal categories.

The localization of $\mathfrak{D}(\operatorname{Ran} X)$ with respect $\mathfrak{D}\left(\operatorname{Ran}^{>n} X\right)$ is equivalent to $\mathfrak{D}\left(\operatorname{Ran}^{\leqslant n} X\right)$; hence, we obtain that $\mathfrak{D}\left(\operatorname{Ran}^{\leqslant n} X\right)$ acquires a canonical symmetric monoidal structure, for which the functors

$$
\mathfrak{D}\left(\operatorname{Ran}^{\leqslant n_{1}} X\right) \longrightarrow \mathfrak{D}\left(\operatorname{Ran}^{\leqslant n_{2}} X\right)
$$

for $n_{1} \geqslant n_{2}$ are symmetric monoidal for the chiral symmetric monoidal structure on $\mathfrak{D}(\operatorname{Ran} X)$.

To establish the pro-nilpotence property of the chiral symmetric monoidal structure on $\mathfrak{D}(\operatorname{Ran} X)$ it suffices to show that the resulting monoidal structure on $\mathfrak{D}\left(\operatorname{Ran}^{\leqslant n} X\right)$ vanishes on

$$
\operatorname{ker}\left(\mathfrak{D}\left(\operatorname{Ran}^{\leqslant n} X\right) \longrightarrow \mathfrak{D}\left(\operatorname{Ran}^{\leqslant n-1} X\right)\right) \otimes \mathfrak{D}\left(\operatorname{Ran}^{\leqslant n} X\right) \longrightarrow \mathfrak{D}\left(\operatorname{Ran}^{\leqslant n} X\right) .
$$

However, the latter is manifest from Lemma 2.3.4.

5.2. Factorization. We shall now prove the second part of the main theorem, that the equivalence between chiral Lie algebras and chiral commutative coalgebras on Ran $X$ interchanges the $\infty$-subcategories of chiral Lie algebras on $X$ and factorization coalgebras.

Theorem 5.2.1. For $A \in \mathrm{Lie}-\operatorname{alg}^{\mathrm{ch}}(\operatorname{Ran} X)$, the corresponding coalgebra $\mathrm{C}^{\mathrm{ch}}(A)$ factorizes if and only if $A$ is supported on $X$, i.e., is an object of Lie-alg ${ }^{\mathrm{ch}}(X)$.

We shall precede the proof of Theorem 5.2.1 by the following two observations made in Sections 5.2 .2 and 5.2 .3 respectively.

5.2.2. Recall that the factorization condition for $B \in \mathrm{Com}^{-\operatorname{coalg}^{\text {ch }}}(\operatorname{Ran} X)$ says that for each surjection $\pi: I \rightarrow J$ the associated map

$$
\jmath(\pi)^{*}\left(\left(\Delta^{I}\right)^{!}(B)\right) \longrightarrow \jmath(\pi)^{*}\left(\underset{j \in J}{\bigotimes}\left(\Delta^{I_{j}}\right)^{!}(B)\right)
$$

is a homotopy equivalence in $\mathfrak{D}(U(\pi))$.

However, we claim that it is enough to check (5.1) for every $I$ and $\pi=\mathrm{id}_{I}$. Indeed, let us assume by induction that the homotopy equivalences (5.1) have been established for finite sets of cardinality $<k$, and let $I$ be with $|I|=k$.

First, we claim that the induction hypothesis implies that (5.1) becomes an ismorphism after applying $\left(\Delta^{\phi}\right)^{!} \circ \jmath(\pi)_{*}$ for any $\phi: I \rightarrow I^{\prime}$ with $\left|I^{\prime}\right|<|I|$. Indeed, set $J^{\prime}:=J \sqcup I_{I}^{\prime}$, and let

$$
\psi: J \rightarrow J^{\prime} \text { and } \phi^{\prime}: I^{\prime} \rightarrow J^{\prime}
$$

denote the corresponding maps. We have:

$$
\left(\Delta^{\phi}\right)^{!} \circ \jmath(\pi)_{*} \simeq \jmath\left(\pi^{\prime}\right)_{*} \circ\left(\Delta^{\psi}\right)^{!}
$$

and thus the situation reduces to that on $X^{I^{\prime}}$.

\footnotetext{
${ }^{9}$ This is a special case of a general notion of ideals of algebras in a pointed monoidal $\infty$-category, where a map $I \rightarrow A$ of nonunital algebras is said to be an ideal if the quotient is equivalent to the quotient as objects, without algebraic structure. For a map $I \rightarrow A$, which is a monomorphism (see [L1, Sect. 5.5.6), this is equivalent to requiring that the resulting maps $I \otimes A \rightarrow A$ and $A \otimes I \rightarrow A$ factor through $I$. We are applying this to the monoidal category $\infty$-Cat pres and a functor $\mathcal{C}^{\prime} \rightarrow \mathcal{C}$ which is fully faithful, which is equivalent to being a monomorphism.
} 
Thus, it is sufficient to show that (5.1) becomes a homotopy equivalence after applying the functor $\jmath\left(\operatorname{id}_{I}\right)^{*} \circ \jmath(\pi)_{*}$. However, in this case the left-hand side becomes $\jmath\left(\operatorname{id}_{I}\right)^{*}\left(\left(\Delta^{I}\right)^{!}(B)\right)$ while the right-hand side becomes

$$
\jmath\left(\operatorname{id}_{I}\right)^{*}\left(\underset{j \in J}{\bigotimes} \jmath\left(\operatorname{id}_{I^{j}}\right)_{*} \circ \jmath\left(\operatorname{id}_{I^{j}}\right)^{*}\left(\Delta^{I_{j}}\right)^{!}(B)\right),
$$

which by the assumption maps isomorphically to

$$
\jmath\left(\operatorname{id}_{I}\right)^{*}\left(\underset{j \in J}{\bigotimes} \jmath\left(\operatorname{id}_{I^{j}}\right)_{*} \circ \jmath\left(\operatorname{id}_{I^{j}}\right)^{*}\left(\left(\left(\Delta^{\text {main }}\right)^{!}(B)\right)^{\bigotimes I^{j}}\right)\right) \simeq \jmath\left(\operatorname{id}_{I}\right)^{*}\left(\left(\left(\Delta^{\text {main }}\right)^{!}(B)\right)^{\bigotimes I}\right) .
$$

Hence, the map in question becomes the map

$$
\jmath\left(\operatorname{id}_{I}\right)^{*}\left(\left(\Delta^{I}\right)^{!}(B)\right) \longrightarrow \jmath\left(\operatorname{id}_{I}\right)^{*}\left(\left(\left(\Delta^{\text {main }}\right) !(B)\right)^{\bigotimes I}\right),
$$

i.e., the map (5.1) for $\pi=\mathrm{id}_{I}$.

5.2.3. The second observation needed for the proof of Theorem 5.2.1 is the canonical filtration on $\mathrm{C}^{\text {ch }}(A)$ as an object of $\mathfrak{D}(\operatorname{Ran} X)$.

Let $\mathcal{C}$ be a stable symmetric monoidal category as in Section 3.2, and let $L$ be an object of Lie-alg $(\mathcal{C})$. By the construction of the Chevalley complex, the object oblv $\left.\operatorname{Com}_{(\mathrm{C}}(L)\right) \in \mathcal{C}$ carries a canonical filtration indexed by positive integers with subquotients described as follows:

$$
\operatorname{gr}^{k}(\mathrm{C}(L)) \simeq \operatorname{Sym}_{\mathcal{C}}^{k}\left(\operatorname{oblv}_{\text {Lie }}(L)[k]\right) .
$$

We will apply it to $\mathcal{C}=\mathfrak{D}(\operatorname{Ran} X)$ equipped with the chiral symmetric monoidal structure.

5.2.4. For future use, let us describe explicitly the object $\operatorname{Sym}^{k, c h}(M) \in \mathfrak{D}(\operatorname{Ran} X)$ for $M \in$ $\mathfrak{D}(\operatorname{Ran} X)$ :

For a finite set $I$, we have

$$
\left(\Delta^{I}\right)^{!}\left(\operatorname{Sym}^{k, \operatorname{ch}}(M)\right) \simeq\left(\underset{\pi: I \rightarrow\{1, \ldots, k\}}{\oplus} \jmath(\pi)_{*} \circ \jmath(\pi)^{*}\left(\underset{j \in\{1, \ldots, k\}}{\bigotimes}\left(\Delta^{I_{j}}\right)^{!}(M)\right)\right)_{\Sigma_{k}} .
$$

Let us consider two particular cases: For $k=1$, we have

$$
\left(\Delta^{I}\right)^{!}\left(\operatorname{Sym}^{1, \operatorname{ch}}(M)\right) \simeq\left(\Delta^{I}\right)^{!}(M) .
$$

Suppose now that $M$ is supported on $X \subset \operatorname{Ran} X$, i.e., if it is of the form $M \simeq\left(\Delta^{\text {main }}\right)_{*}\left(M_{X}\right)$ for some $M_{X} \in \mathfrak{D}(X)$. We have that $\left(\Delta^{I}\right)^{!}\left(\operatorname{Sym}^{k, \mathrm{ch}}(M)\right)$ is zero unless $|I|=k$, and for $I$ with $|I|=k$

$$
\left(\Delta^{I}\right)^{!}\left(\operatorname{Sym}^{k, \operatorname{ch}}(M)\right) \simeq \jmath\left(\operatorname{id}_{I}\right)_{*} \circ \jmath\left(\operatorname{id}_{I}\right)^{*}\left(M_{X}^{\bowtie I}\right) .
$$

5.2.5. Proof of Theorem 5.2.1, the "if" direction. Let us first show that if $A$ is supported on $X$, then $\mathrm{C}^{\mathrm{ch}}(A)$ factorizes. By Section 5.2.2 we need to show that the map

$$
\jmath\left(\operatorname{id}_{I}\right)^{*}\left(\left(\Delta^{I}\right)^{!}\left(\mathrm{C}^{\mathrm{ch}}(A)\right)\right) \longrightarrow \jmath\left(\operatorname{id}_{I}\right)^{*}\left(\left(\left(\Delta^{\text {main }}\right) !\left(\mathrm{C}^{\mathrm{ch}}(A)\right)\right)^{凶 I}\right)
$$

is a homotopy equivalence.

Let us denote by $A_{X}$ the D-module on $X$ such that $A=\left(\Delta^{\text {main }}\right)_{*}\left(A_{X}\right)$. Consider the canonical filtration on oblv ${ }_{\mathrm{Com}}^{\mathrm{ch}}\left(\mathrm{C}^{\mathrm{ch}}(A)\right)$ of Section 5.2.3.

We obtain that the functor $\jmath\left(\operatorname{id}_{I}\right)^{*}$ annihilates all $\operatorname{gr}^{k}\left(\left(\Delta^{I}\right)^{!}\left(\mathrm{C}^{\mathrm{ch}}(A)\right)\right)$ except for one with $k=|I|$, and in the latter case we have

$$
\jmath\left(\operatorname{id}_{I}\right)^{*}\left(\operatorname{gr}^{k}\left(\left(\Delta^{I}\right)^{!}\left(\mathrm{C}^{\mathrm{ch}}(A)\right)\right)\right) \simeq \jmath\left(\operatorname{id}_{I}\right)^{*}\left(A_{X}^{凶 I}[|I|]\right) .
$$

(In particular, for $k=1$, the map $A_{X}[1] \longrightarrow\left(\Delta^{\text {main }}\right)^{!}\left(\mathrm{C}^{\text {ch }}(A)\right)$ is a homotopy equivalence.) 
Under these identifications, the map of (5.2) becomes the map

$$
\jmath\left(\operatorname{id}_{I}\right)^{*}\left(\left(\Delta^{I}\right)^{!}\left(\mathrm{C}^{\mathrm{ch}}(A)\right)\right) \simeq \jmath\left(\operatorname{id}_{I}\right)^{*}\left(\operatorname{gr}^{k}\left(\left(\Delta^{I}\right)^{!}\left(\mathrm{C}^{\mathrm{ch}}(A)\right)\right)\right) \simeq \jmath\left(\operatorname{id}_{I}\right)^{*}\left(A_{X}^{\llbracket I I}[|I|]\right) .
$$

5.2.6. Proof of Theorem 5.2.1, the "only if" direction. Let us now prove the implication in the opposite direction. Assume that $A \in \mathrm{Lie}_{-} \operatorname{alg}^{\mathrm{ch}}(\operatorname{Ran} X)$ is such that the underlying D-module is not supported on $X$. Let us show that $\mathrm{C}^{\mathrm{ch}}(A)$ does not factorize.

By assumption, there exists a finite set $I$ with $|I| \geqslant 2$, such that $\jmath\left(\operatorname{id}_{I}\right)^{*}\left(\left(\Delta^{I}\right)^{!}(A)\right) \neq 0$. Let us take $I$ to be of minimal cardinality among such.

Consider the canonical filtration on $\left(\Delta^{I}\right)^{!}\left(\mathrm{C}^{\mathrm{ch}}(A)\right)$, and the induced filtration on

$$
\jmath\left(\operatorname{id}_{I}\right)^{*}\left(\left(\Delta^{I}\right)^{!}\left(\mathrm{C}^{\mathrm{ch}}(A)\right)\right) \text {. }
$$

By assumption, the only non-vanishing terms of $\jmath\left(\operatorname{id}_{I}\right)^{*}\left(\operatorname{gr}^{k}\left(\left(\Delta^{I}\right)^{!}\left(\mathrm{C}^{\mathrm{ch}}(A)\right)\right)\right)$ occur for $k=1$ and $k=|I|$ with the former being canonically isomorphic to $f\left(\operatorname{id}_{I}\right)^{*}\left(\left(\Delta^{I}\right)^{!}(A)\right)$, and the latter to

$$
\jmath\left(\operatorname{id}_{I}\right)^{*}\left(\left(\left(\Delta^{\text {main }}\right)^{!}\left(\mathrm{C}^{\mathrm{ch}}(A)\right)\right)^{\bowtie I}\right) .
$$

The map (5.2), identifies with the map

$$
\jmath\left(\operatorname{id}_{I}\right)^{*}\left(\left(\Delta^{I}\right)^{!}\left(\mathrm{C}^{\mathrm{ch}}(A)\right)\right) \rightarrow \jmath\left(\operatorname{id}_{I}\right)^{*}\left(\operatorname{gr}^{k}\left(\left(\Delta^{I}\right)^{!}\left(\mathrm{C}^{\mathrm{ch}}(A)\right)\right)\right),
$$

which is not a homotopy equivalence, since it annihilates the first term of the filtration.

\section{Chiral envelopes of $\star$-Lie algebras}

\subsection{The basic commutative diagram.}

6.1.1. By construction, we have a natural map $\otimes^{\star} \rightarrow \otimes^{\text {ch }}$ between the two symmetric monoidal structures on $\mathfrak{D}(\operatorname{Ran} X)$. More precisely, the identity functor on $\mathfrak{D}(\operatorname{Ran} X)$ is a left lax symmetric monoidal structure, when viewed as a functor from $\mathfrak{D}(\operatorname{Ran} X)$ equipped with the $\star$ symmetric monoidal structure to $\mathfrak{D}(\operatorname{Ran} X)$ equipped with the chiral monoidal structure.

For an operad $\mathcal{O}$ (resp., cooperad $\mathcal{P}$ ) we let $\operatorname{oblv}_{\mathcal{O}}^{\mathrm{ch} \rightarrow \star}\left(\right.$ resp., oblv $\left.\mathcal{P}^{\star \rightarrow \mathrm{ch}}\right)$ denote the corresponding forgetful functors

$$
\mathcal{O}-\operatorname{alg}^{\operatorname{ch}}(\operatorname{Ran} X) \rightarrow \mathcal{O}-\operatorname{alg}^{\star}(\operatorname{Ran} X) \text { and } \mathcal{P} \text {-coalg }{ }^{\star}(\operatorname{Ran} X) \rightarrow \mathcal{P} \text {-coalg }{ }^{\operatorname{ch}}(\operatorname{Ran} X) .
$$

Both of these functors commute with the forgetful functors to $\mathfrak{D}(\operatorname{Ran} X)$.

In particular, we obtain a natural forgetful functor

$$
\text { oblv }_{\text {Lie }}^{\text {ch } \rightarrow \star}: \text { Lie-alg }{ }^{\text {ch }}(\operatorname{Ran} X) \rightarrow \operatorname{Lie}-\operatorname{alg}^{\star}(\operatorname{Ran} X) .
$$

The above functor is easily seen to commute with limits (since on both sides the forgetful functor to $\mathfrak{D}(\operatorname{Ran} X)$ is conservative and commutes with limits). Since the categories involved are presentable, we obtain that the functor in (6.1) admits a left adjoint. We denote the resulting left adjoint functor

$$
\operatorname{Lie}-\operatorname{alg}^{\star}(\operatorname{Ran} X) \longrightarrow \text { Lie-alg }{ }^{\operatorname{ch}}(\operatorname{Ran} X)
$$

by $\operatorname{Ind}_{\mathrm{Lie}}^{\star \rightarrow \mathrm{ch}}$.

Our basic observation is the following: 
Proposition 6.1.2. We have a commutative diagram of functors

$$
\begin{aligned}
& \text { Lie-alg }{ }^{\text {ch }}(\operatorname{Ran} X) \stackrel{\mathrm{C}^{\mathrm{ch}}}{\longrightarrow} \text { Com-coalg }{ }^{\mathrm{ch}}(\operatorname{Ran} X) \\
& \operatorname{Ind}_{\mathrm{Lie}}^{\star \rightarrow \mathrm{ch}} \uparrow \quad \uparrow \mathrm{oblv}_{\mathrm{Com}}^{\star \rightarrow \mathrm{ch}} \\
& \text { Lie-alg }{ }^{\star}(\operatorname{Ran} X) \stackrel{\mathrm{C}^{\star}}{\longrightarrow} \operatorname{Com}^{-\operatorname{coalg}^{\star}}(\operatorname{Ran} X)
\end{aligned}
$$

6.2. Recollections on monads. For the proof of Proposition 6.1.2 we need to recall several facts about calculus of monads. The general reference for this material is [L2, Sect. 6.2.

6.2.1. Recall that for a category $\mathcal{C}$, a monad $M$ acting on $\mathcal{C}$ is, by definition, a unital associative algebra in the monoidal category $\operatorname{Funct}(\mathcal{C}, \mathcal{C})$ of endo-functors on $\mathcal{C}$.

The monoidal category Funct $(\mathcal{C}, \mathcal{C})$ acts on $\mathcal{C}$, so it makes sense to talk about $M$-modules in $\mathcal{C}$; we denote this category by $\operatorname{Mod}_{M}$. We shall denote by oblv $M$ the forgetful functor $\operatorname{Mod}_{M} \rightarrow \mathcal{C}$, and by $\operatorname{Ind}_{M}$ its left adjoint.

Let $F: \mathcal{C} \rightarrow \mathcal{D}$ (resp., $G: \mathcal{D} \rightarrow \mathcal{C}$ ) be a functor. There is a natural notion of right (resp., left) action of a monad $M$ on $F$ (resp., $G$ ): We view $\operatorname{Funct}(\mathcal{C}, \mathcal{D})$ (resp., $\operatorname{Funct}(\mathcal{D}, \mathcal{C})$ ) as a right (resp., left) module over Funct $(\mathcal{C}, \mathcal{C})$.

If $G$ is the right adjoint of $F$, then the data of action of $M$ on $F$ is equivalent to that of action of $M$ on $G$.

Moreover, a datum of action of $M$ on $G$ is equivalent to factoring $G$ as a composition

$$
\mathcal{D} \stackrel{G^{\prime}}{\rightarrow} \operatorname{Mod}_{M} \stackrel{\text { oblv }_{M}}{\longrightarrow} \mathcal{C} .
$$

Similarly, a datum of action of $M$ on $F$ is equivalent to factoring $F$ as a composition

$$
\mathcal{C} \stackrel{\operatorname{Ind}_{M}}{\longrightarrow} \operatorname{Mod}_{M} \stackrel{F^{\prime}}{\rightarrow} \mathcal{D} .
$$

6.2.2. For an adjoint pair

$$
F: \mathcal{C} \rightleftarrows \mathcal{D}: G
$$

as above, there exists a universal monad on $\mathcal{C}$ that acts on $F$ (or, equivalently, on $G$ ). As a plain endo-functor on $\mathcal{C}$, this monad is isomorphic to $G \circ F$. Thus, we can view this construction as endowing $G \circ F$ with a structure of monad.

By the universal property, a datum of action of a $\operatorname{monad} M$ on $F$ (resp., $G$ ) is equivalent to that of homomorphism of monads $M \rightarrow G \circ F$.

By Section 6.2.1 the identity map on the monad $G \circ F$ yields a canonical factorization of the functor $G$ as

$$
\mathcal{D} \stackrel{G^{\mathrm{enh}}}{\longrightarrow} \operatorname{Mod}_{G \circ F} \stackrel{\text { oblv }_{G \circ F}}{\longrightarrow} \mathcal{C} .
$$

Thus, we can view the category $\operatorname{Mod}_{G \circ F}$ as "the best approximation" to $\mathcal{D}$ from the point of view of $\mathcal{C}$.

For the sake of completeness, let us also mention that the Barr-Beck-Lurie theorem gives a necessary and sufficient condition on the functor $G$, for the resulting functor $G^{\text {enh }}$ to be an equivalence. 


\subsubsection{Let}

$$
F: \mathcal{C} \rightleftarrows \mathcal{D}: G
$$

be as above, and let $M_{\mathcal{D}}$ be a monad on $\mathcal{D}$. We can view the functor $G \circ M_{\mathcal{D}} \circ F$ as the composition of $\operatorname{Ind}_{M_{\mathcal{D}}} \circ F$ with its right adjoint. Hence, the above procedure endows $G \circ M_{\mathcal{D}} \circ F$ with a structure of monad.

If $M_{\mathcal{C}}$ is a monad on $\mathcal{C}$, a datum of homomorphism $M_{\mathcal{C}} \rightarrow G \circ M_{\mathcal{D}} \circ F$ is equivalent to a datum of action of $M_{\mathcal{C}}$ on the composition $G \circ$ oblv $_{M_{\mathcal{D}}}$, and hence to that of a commutative diagram

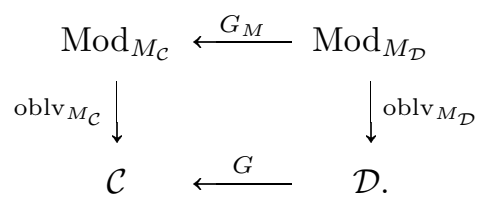

Under such circumstances, we shall denote by $\operatorname{Ind}_{M}^{F}$ the left adjoint of $G_{M}$, which makes the following diagram commutative:

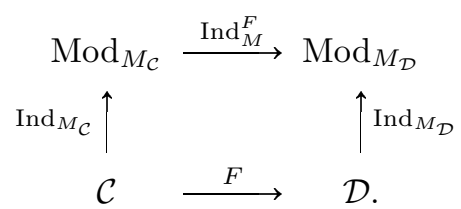

6.2.4. The above facts render to the world of comonads by reversing the arrows.

6.2.5. Let $F: \mathcal{C} \rightleftarrows \mathcal{D}: G, M_{\mathcal{C}}$ and $M_{\mathcal{D}}$ be as above. Assume now that both $M_{\mathcal{C}}$ and $M_{\mathcal{D}}$ are augmented, and assume that the datum of homomorphism $M_{\mathcal{C}} \rightarrow G \circ M_{\mathcal{D}} \circ F$ is compatible with the augmentations. This equivalent to extending the diagram (6.3) to a commutative diagram

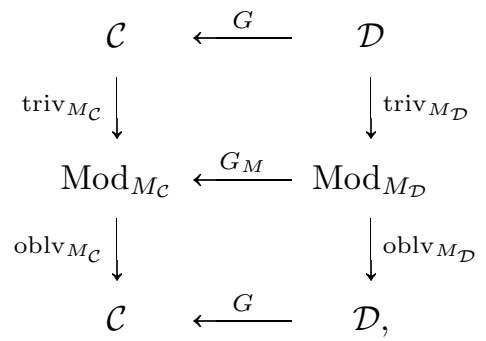

where $\operatorname{triv}_{M_{\mathcal{C}}}$ (resp., $\operatorname{triv}_{M_{\mathcal{D}}}$ ) is the functor corresponding to the augmentation on $M_{\mathcal{C}}$ (resp., $M_{\mathcal{D}}$ ).

Let $N_{\mathcal{C}}$ be the Koszul dual comonad, i.e., the one corresponding to the adjoint pair of functors

$$
\operatorname{Bar}_{M_{\mathcal{C}}}: \operatorname{Mod}_{M_{\mathcal{C}}} \rightleftarrows \mathcal{C}: \operatorname{triv}_{M_{\mathcal{C}}} .
$$

By Section 6.2.2, the functor $\operatorname{Bar}_{M_{\mathcal{C}}}$ canonically factors as

$$
\operatorname{Mod}_{M_{\mathcal{C}}} \stackrel{\operatorname{Bar}_{M_{\mathcal{C}}}^{\mathrm{enh}}}{\longrightarrow} \operatorname{Comod}_{N_{\mathcal{C}}} \stackrel{\operatorname{oblv}_{N_{\mathcal{C}}}}{\longrightarrow} \mathcal{C},
$$

and similarly for the monad $M_{\mathcal{D}}$ acting on $\mathcal{D}$.

We claim that we have a natural homomorphism of comonads $F \circ N_{\mathcal{C}} \circ G \rightarrow N_{\mathcal{D}}$. Indeed, defining such homomorphism is equivalent to making the comonad $N_{\mathcal{D}}$ coact on the functor $\operatorname{triv}_{M_{\mathcal{C}}} \circ G$. However, the latter functor is isomorphic to $G_{M} \circ \operatorname{triv}_{M_{\mathcal{D}}}$, and $\operatorname{triv}_{M_{\mathcal{D}}}$ is canonically coacted on by $N_{\mathcal{D}}$.

Thus, we obtain a commutative diagram of functors

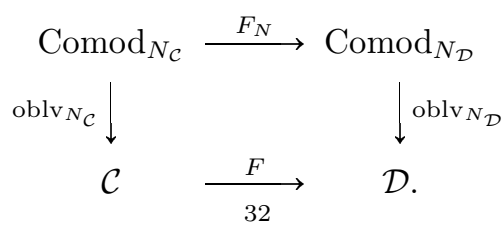


In the above circumstances, we claim:

Lemma 6.2.6. The following diagram of functors canonically commutes:

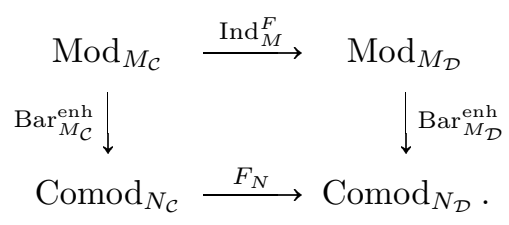

Proof. The diagram

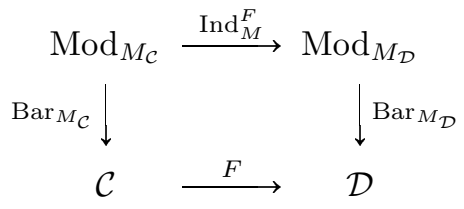

naturally commutes, being obtained from the top square in (6.4), i.e.,

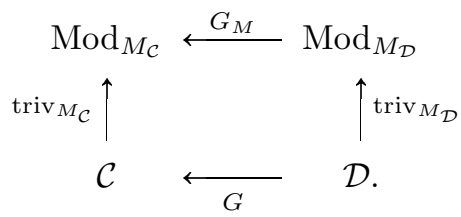

by taking the left adjoints.

Thus, we need to show that the two coactions of $N_{\mathcal{D}}$ on the resulting functor

$$
\operatorname{Mod}_{M_{\mathcal{C}}} \rightarrow \mathcal{D}
$$

corresponding to the two circuits in the diagram (6.5) are homotopy equivalent. This is, in turn, equivalent to showing that the the two coactions on the composed functor $\mathcal{D} \rightarrow \operatorname{Mod}_{M_{\mathcal{C}}}$ in (6.6) are homotopy equivalent. However, the latter follows from the construction of the homomorphism of comonads $F \circ N_{\mathcal{C}} \circ G \rightarrow N_{\mathcal{D}}$.

6.2.7. Proof of Proposition 6.1.2, To prove Proposition 6.1.2, we apply Lemma 6.2.6 to $\mathcal{C}=\mathcal{D}=$ $\mathfrak{D}(\operatorname{Ran} X)$ with $M_{\mathcal{C}}$ being the monad $\mathrm{T}_{\text {Lie }}^{\star}$ and $M_{\mathcal{D}}$ being the monad $\mathrm{T}_{\text {Lie }}^{\mathrm{ch}}$, and $F$ being the identity functor.

\subsection{Chiral homology of chiral envelopes.}

6.3.1. Let $f: X \rightarrow Y$ be a map of schemes. The presentation of $\mathfrak{D}(\operatorname{Ran} X)$ as in (2.3) defines a functor

$$
\left(f^{\operatorname{Ran}}\right)_{*}: \mathfrak{D}(\operatorname{Ran} X) \longrightarrow \mathfrak{D}(\operatorname{Ran} Y),
$$

via $\left(f^{I}\right)_{*}: \mathfrak{D}\left(X^{I}\right) \rightarrow \mathfrak{D}\left(Y^{I}\right)$ for $I \in \mathrm{fSet}^{\text {surj }}$. The next lemma results from the definitions:

Lemma 6.3.2. The functor $\left(f^{\text {Ran }}\right)_{*}$ has a natural symmetric monoidal functor with respect to the

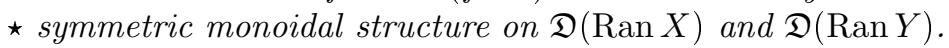

6.3.3. Let us take in the previous setup $Y=$ pt. We shall denote the resulting symmetric monoidal functor $\mathfrak{D}(\operatorname{Ran} X) \rightarrow \operatorname{Vect}_{k}$ by

$$
\Gamma_{\mathrm{DR}}(\operatorname{Ran} X,-) \text {. }
$$

Being symmetric monoidal, this functor gives rise to a functor

$$
\Gamma_{\mathrm{DR}}(\operatorname{Ran} X,-)_{\mathcal{O}}: \mathcal{O}-\operatorname{alg}(\mathfrak{D}(\operatorname{Ran} X)) \rightarrow \mathcal{O}-\operatorname{alg}\left(\operatorname{Vect}_{k}\right)
$$

for any operad $\mathcal{O}$ and

$$
\Gamma_{\text {DR }}(\operatorname{Ran} X,-)_{\mathcal{P}}: \mathcal{P}-\operatorname{coalg}(\mathfrak{D}(\operatorname{Ran} X)) \rightarrow \mathcal{P}-\text { coalg }_{\left(\operatorname{Vect}_{k}\right)}
$$


for any cooperad $\mathcal{P}$.

6.3.4. Let us recall from [BD1, Sect. 4.2, that the functor of chiral homology

$$
\int_{X}: \text { Lie-alg }{ }^{\text {ch }}(\operatorname{Ran} X) \rightarrow \text { Vect }_{k}
$$

is by definition the composition

$$
\text { Lie-alg }{ }^{\text {ch }}(\operatorname{Ran} X) \stackrel{\mathrm{C}^{\mathrm{ch}}}{\longrightarrow} \mathrm{Com}-\operatorname{coalg}^{\mathrm{ch}}(\operatorname{Ran} X) \stackrel{\mathrm{oblv}_{\mathrm{Com}}^{\mathrm{ch}}}{\longrightarrow} \mathfrak{D}(\operatorname{Ran} X) \stackrel{\Gamma_{\mathrm{DR}}(\operatorname{Ran} X,-)}{\longrightarrow} \operatorname{Vect}_{k} .
$$

6.3.5. We shall now prove the following:

Proposition 6.3.6. The following diagram of functors

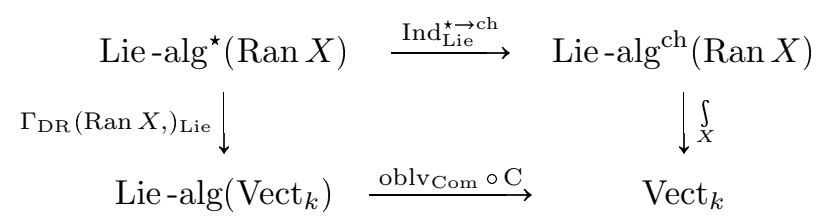

is canonically commutative.

Proof. First, applying Proposition 6.1.2, we rewrite the composition

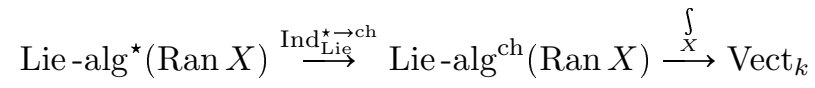

as

$$
\text { Lie-alg }{ }^{\star}(\operatorname{Ran} X) \stackrel{\mathrm{C}^{\star}}{\longrightarrow} \mathrm{Com}-\operatorname{coalg}^{\star}(\mathfrak{D}(\operatorname{Ran} X)) \stackrel{\mathrm{oblv}_{\mathrm{C} \text { om }}^{\star}}{\longrightarrow} \mathfrak{D}(\operatorname{Ran} X) \stackrel{\Gamma_{\mathrm{DR}}(\operatorname{Ran} X,-)}{\rightarrow} \operatorname{Vect}_{k},
$$

and further as

$$
\operatorname{Lie}_{-a \lg ^{\star}}(\operatorname{Ran} X) \stackrel{\mathrm{C}^{\star}}{\longrightarrow} \operatorname{Com}-\operatorname{coalg}^{\star}(\mathfrak{D}(\operatorname{Ran} X)) \stackrel{\Gamma_{\mathrm{DR}}(\operatorname{Ran} X,-)_{\mathrm{Com}}}{\longrightarrow} \mathrm{Com}-\operatorname{coalg}\left(\operatorname{Vect}_{k}\right) \stackrel{\text { oblveom }^{\longrightarrow}}{\longrightarrow} \operatorname{Vect}_{k} .
$$

Hence, to prove the proposition, it suffices to show that the following diagram of functors is commutative:

$$
\begin{array}{ccc}
\operatorname{Lie}_{-\operatorname{alg}^{\star}}(\operatorname{Ran} X) & \stackrel{\mathrm{C}^{\star}}{\longrightarrow} & \operatorname{Com}-\operatorname{coalg}^{\star}(\mathfrak{D}(\operatorname{Ran} X)) \\
\Gamma_{\mathrm{DR}}(\operatorname{Ran} X,-)_{\mathrm{Lie}} \downarrow & & \\
\operatorname{Lie}-\operatorname{alg}\left(\operatorname{Vect}_{k}\right) & \mathrm{C} & \text { Com }-\operatorname{coalg}\left(\operatorname{Vect}_{k}\right) .
\end{array}
$$

However, this follows from Lemma 6.2.6.

We apply this lemma it to $\mathcal{C}=\mathfrak{D}(\operatorname{Ran} X), \mathcal{D}=\operatorname{Vect}_{k}, M_{\mathcal{C}}=\mathrm{T}_{\text {Lie }}^{\star}, M_{\mathcal{D}}=\mathrm{T}_{\text {Lie }}$, and $F=$ $\Gamma_{\mathrm{DR}}(\operatorname{Ran} X,-)$. Note that the functor $\operatorname{Ind}_{M}^{F}$ of Lemma 6.2.6 is isomorphic in our case to just $\Gamma_{\mathrm{DR}}(\operatorname{Ran} X,-)_{\mathrm{Lie}}$, since $\Gamma_{\mathrm{DR}}(\operatorname{Ran} X,-)$ is monoidal and not just left lax monoidal.

\subsection{Chiral envelopes and factorization.}

6.4.1. Our current goal is to prove the following:

Theorem 6.4.2. The functor $\operatorname{Ind}_{\mathrm{Lie}}^{\star \rightarrow \mathrm{ch}}$ sends the subcategory

$$
\operatorname{Lie}-\operatorname{alg}^{\star}(X) \subset \operatorname{Lie}-\operatorname{alg}^{\star}(\operatorname{Ran} X)
$$

to the subcategory

$$
\text { Lie-alg }{ }^{\text {ch }}(X) \subset \text { Lie-alg }^{\text {ch }}(\operatorname{Ran} X) .
$$

Before we prove Theorem 6.4.2 let us derive some corollaries: 
Corollary 6.4.3. The resulting functor

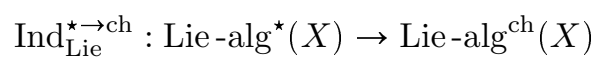

is the left adjoint of the forgetful functor

$$
\text { Lie-alg }{ }^{\star}(X) \leftarrow \text { Lie-alg }{ }^{c h}(X): \operatorname{oblv}_{\text {Lie }}^{\text {ch } \rightarrow \star} .
$$

We shall sometimes use the notation $U^{\text {ch }}$ for the functor in (6.7). This is the higher-dimensional and derived version of the chiral enveloping functor of BD1, Sect. 3.7.

From Proposition 6.3.6 we obtain:

Corollary 6.4.4. For $L \in \mathrm{Lie}_{-} \operatorname{alg}^{\star}(X)$ there exists a canonical homotopy equivalence

$$
\int_{X} U^{\mathrm{ch}}(L) \simeq \operatorname{oblv}_{\mathrm{Com}} \circ \mathrm{C}\left(\Gamma_{\mathrm{DR}}(\operatorname{Ran} X, L)_{\text {Lie }}\right) .
$$

Remark 6.4.5. In the situation of the above corollary, let $L_{X}$ be the D-module on $X$, such that

$$
\left(\Delta^{\text {main }}\right)_{*}\left(L_{X}\right) \simeq \operatorname{oblv}_{\text {Lie }}^{\star}(L)
$$

Note that

$$
\operatorname{oblv}_{\text {Lie }}\left(\Gamma_{\mathrm{DR}}(\operatorname{Ran} X, L)_{\mathrm{Lie}}\right) \simeq \Gamma_{\mathrm{DR}}\left(X, L_{X}\right)
$$

which gives the object $\Gamma_{\mathrm{DR}}\left(X, L_{X}\right) \in \operatorname{Vect}_{k}$ a canonical Lie algebra structure. Thus, Corollary 6.4.4 gives a conceptual proof of (a generalization of) a theorem from [BD1, Sect. 4.8.1 that computes the chiral homology of chiral envelopes of $\star$-Lie algebras.

Remark 6.4.6. The actual theorem of [BD1] is slightly different from ours. Namely, in loc.cit. one considers the unital version of $U^{\mathrm{ch}}(L)$, and proves the result about its chiral homology. Thus, in order to obtain their formulation one needs to complement Corollary 6.4.4 by one more theorem that shows that chiral homology of a non-unital chiral Lie algebra $A$ differs from the chiral homology of the corresponding unital chiral Lie algebra by a copy of the ground field $k$, provided that $X$ is connected; see loc.cit., Proposition 4.4.8.

Remark 6.4.7. Note that Theorem 6.4.2 allows to construct non-commutative chiral Lie algebras on $X$, for $X$ of any dimension: start with a $\star$-Lie algebra $L$ and take $U^{\text {ch }}(L)$.

For example, let $L^{\prime}$ be a Lie algebra in Vect ${ }_{k}$. Then the D-module $L:=\underline{L^{\prime}}$ on $X$, corresponding to the "constant sheaf" with fiber $L^{\prime}$, is naturally a $\star$-Lie algebra on $X$. Thus, for any $L^{\prime}$ as above, we can produce the chiral Lie algebra $U^{\text {ch }}\left(\underline{L^{\prime}}\right)$.

As another example, we can take $L=L^{\prime} \otimes D_{X}$, where $D_{X} \in \mathfrak{D}(X)$ is the ring of differential operators. The structure of $\star$-Lie algebra on $L^{\prime} \otimes D_{X}$ is defined as in [BD1], Example 2.5.6(b)(ii). Or we can consider $L=\Theta_{X} \underset{\mathcal{O}_{X}}{\otimes} D_{X}$, where $\Theta_{X}$ is the algebroid of vector fields on $X$, see [BD1], Example 2.5.6(b)(i) 10

Note, however, that by Remark 6.5.4 unless $\operatorname{dim}(X)=1$, if we start with $L$ which lies in the heart of the natural t-structure on $X$ and is flat as a quasi-coherent sheaf, the chiral Lie algebra $U^{\text {ch }}(L)$ considered as a D-module on $X$, will not lie in the heart of the t-structure. This is closely analogous to the topological setting: For $n \geqslant 2$, any $\mathcal{E}_{n}$-algebra over a field of characteristic zero that lies in the heart of the t-structure on chain complexes (i.e., is discrete) has a commutative algebra structure.

By the same remark, if we want to obtain $U^{\text {ch }}(L)$, which up to a cohomological shift, lies in the heart of the t-structure, we typically need to start with $L$, such that $L[1-\operatorname{dim}(X)]$ lies in the heart of the t-structure. However, the $\star$-Lie algebra structure on such $L$ is automatically trivial, unless

\footnotetext{
${ }^{10}$ The question of constructing central extensions of these examples à la Kac-Moody or Virasoro is much more subtle.
} 
$\operatorname{dim}(X)=1$. Likewise, in the topological setting, the $\mathcal{E}_{n}$-enveloping algebra of a Lie algebra never lies in the heart of the t-structure, for $n \geqslant 2$.

To summarize: In higher dimensions, it is difficult to produce non-commutative chiral Lie algebras that lie in the heart of the t-structure on $\mathfrak{D}(X)$.

6.4.8. For the proof of Theorem 6.4.2 let us recall the setting of Section [5.2.3. We shall need one more property of this construction, which we shall state in a form which is somewhat crude, but will suffice for our purposes.

Let $\mathcal{C}$ and $L$ be as in Section 5.2 .3 For a positive integer $k$ let $\mathrm{C}(L) \leqslant k$ denote the corresponding term of the filtration on oblv $\operatorname{Com}_{(\mathrm{C}}(\mathrm{C}(L))$. We claim that the coalgebra structure on $\mathrm{C}(L)$ is compatible with the filtration in the following weak sense:

For positive integers $k$ and $n$ and a partition $k=k_{1}+\cdots+k_{n}$ we have a map

$$
\mathrm{C}(L)_{\leqslant k} \rightarrow \mathrm{C}(L)_{\leqslant k_{1}} \otimes \ldots \otimes \mathrm{C}(L)_{\leqslant k_{n}}
$$

satisfying the natural associativity property. For $k^{\prime} \geqslant k$ and $k_{i}^{\prime} \geqslant k_{i}, i=1, \ldots, n$ the diagram

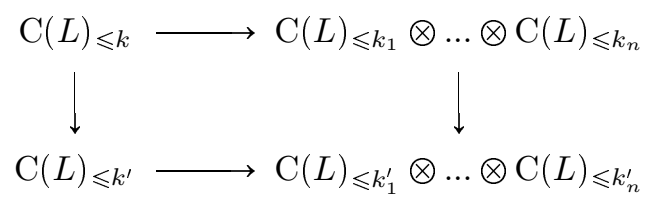

is commutative, and the diagram

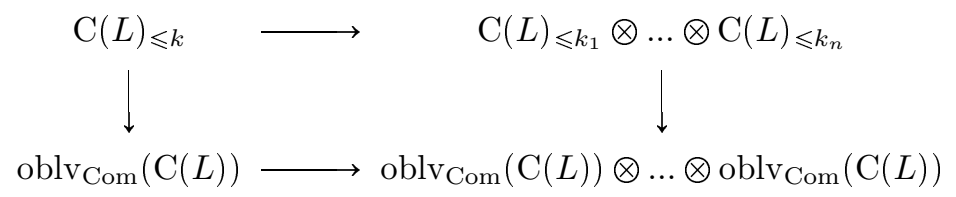

is commutative as well. In particular, for $k$ and $n$ as above, we obtain the maps

$$
\operatorname{gr}^{k}(\mathrm{C}(L)) \rightarrow \operatorname{gr}^{k_{1}}(\mathrm{C}(L)) \otimes \ldots \otimes \operatorname{gr}^{k_{n}}(\mathrm{C}(L)),
$$

that also have the natural associativity property.

The final property that we need is the following:

Recall the identification

$$
\operatorname{gr}^{k}(\mathrm{C}(L)) \simeq \operatorname{Sym}_{\mathcal{C}}^{k}(L) \simeq \operatorname{gr}^{k}\left(\mathrm{C}\left(L_{\text {triv }}\right)\right),
$$

where $L_{\text {triv }}:=\operatorname{triv}_{\text {Lie }} \circ \operatorname{oblv}_{\text {Lie }}(L)$. We obtain that the diagrams

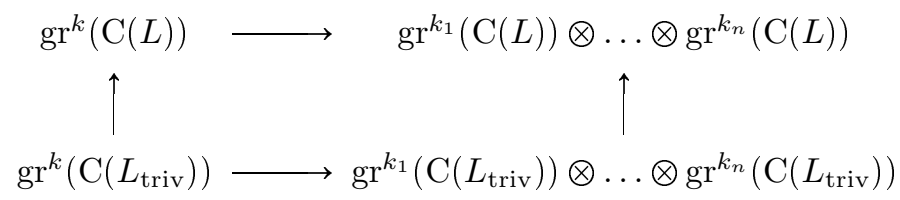

commute, where in the upper horizontal row we use the map (6.8), and in the lower horizontal row the map is (6.8) for $L_{\text {triv }}$.

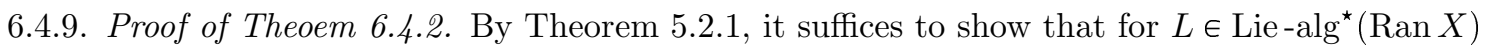

$$
B:=\mathrm{C}^{\mathrm{ch}}\left(\operatorname{Ind}_{\mathrm{Lie}}^{\star \rightarrow \mathrm{ch}}(L)\right) \in \mathrm{Com}-\operatorname{coalg}^{\mathrm{ch}}(\operatorname{Ran} X)
$$

factorizes. We will use the discussion in Section 5.2.2 and show that for every finite set $I$ the corresponding map

$$
\jmath\left(\operatorname{id}_{I}\right)^{*}\left(\left(\Delta^{I}\right)^{!}(B)\right) \rightarrow \jmath\left(\operatorname{id}_{I}\right)^{*}\left(\left(\left(\Delta^{\text {main }}\right)^{!}(B)\right)^{\bigotimes I}\right)
$$

is a homotopy equivalence in $\mathfrak{D}\left(U\left(\mathrm{id}_{I}\right)\right)$. 
By Proposition 6.1.2, we have:

$$
B \simeq \operatorname{oblv}_{\mathrm{Com}}^{\star \rightarrow \operatorname{ch}}\left(\mathrm{C}^{\star}(L)\right)
$$

Consider now the filtration on both sides of (6.10) given by the filtration on

$$
\operatorname{oblv}_{\mathrm{Com}}^{\star}\left(\mathrm{C}^{\star}(L)\right)
$$

as in Section 6.4.8.

We obtain that it is sufficient to show that the maps

$$
\operatorname{gr}^{\bullet}(B) \rightarrow \underset{I}{\underset{\otimes}{\star}} \operatorname{gr}^{\bullet}(B)
$$

of (6.8) become homotopy equivalences after applying $\jmath\left(\operatorname{id}_{I}\right)^{*} \circ\left(\Delta^{I}\right)$ ! . However, (6.9) allows to reduce the latter assertion to the case when $L$ has the trivial Lie algebra structure.

Thus, we have to show that for $M \in \mathfrak{D}(\operatorname{Ran} X)$ of the form $\left(\Delta^{\text {main }}\right)_{*}\left(M_{X}\right)$, and the coalgebra

$$
B:=\operatorname{Sym}^{\bullet}{ }^{\star}(M),
$$

the maps (6.10) are homotopy equivalences.

However, it is easy to see that

$$
\jmath\left(\operatorname{id}_{I}\right)^{*} \circ\left(\Delta^{I}\right)^{!}\left(\operatorname{Sym}^{n, \star}(M)\right) \simeq \underset{n=\sum_{i \in I} n_{i}}{ } \jmath\left(\operatorname{id}_{I}\right)^{*}\left(\underset{i}{\bowtie} \operatorname{Sym}^{n_{i}, !}\left(M_{X}\right)\right),
$$

where $\operatorname{Sym}^{n_{i}, !}\left(M_{X}\right)$ denotes the corresponding symmetric power taken in category $\mathfrak{D}(X)$, with respect to the symmetric monoidal structure given by tensor product (see Section 1.4.7). This makes the homotopy equivalence (6.10) for $\operatorname{Sym}^{\bullet, \star}(M)$ manifest.

\subsection{The Poincaré-Birkhoff-Witt theorem.}

6.5.1. We shall now use Theorem 6.4.2 and Proposition 6.1.2 to prove a generalized version of the PBW theorem of chiral universal enveloping algebras, stated in the original form as Theorem 3.7.14 of [BD1].

Thus, let $L$ be a $\star$-Lie algebra on $X$, and let $U^{\text {ch }}(L) \in$ Lie-alg $^{\text {ch }}(X)$ be its chiral universal enveloping algebra. Let $U^{\mathrm{ch}}(L)_{X}$ denote the corresponding object of $\mathfrak{D}(X)$.

By Theorem 6.4.2 and Proposition 6.1.2, we have a homotopy equivalence of D-modules on $X$ :

$$
U^{\mathrm{ch}}(L)_{X} \simeq\left(\Delta^{\text {main }}\right)^{!}\left(\operatorname{oblv}_{\mathrm{Com}}^{\star}\left(\mathrm{C}^{\star}(L)\right)\right)[-1]
$$

The filtration of Section 5.2 .3 on $\operatorname{oblv}_{\mathrm{Com}}^{\star}\left(\mathrm{C}^{\star}(L)\right)$ defines a filtration on $U^{\mathrm{ch}}(L)_{X}$.

Corollary 6.5.2. The associated graded $\operatorname{gr}^{\bullet}\left(U^{\mathrm{ch}}(L)_{X}\right)$ is canonically isomorphic to

$$
\operatorname{Sym}^{\bullet, !}\left(L_{X}[1]\right)[-1] \text {, }
$$

where $\left(\Delta^{\text {main }}\right)_{*}\left(L_{X}\right) \simeq L$.

6.5.3. Proof of Corollary 6.5.2. The proof follows immediately from the homotopy equivalences

$$
\operatorname{gr}^{\bullet}\left(\operatorname{oblv}_{\mathrm{Com}}^{\star}\left(\mathrm{C}^{\star}(L)\right)\right) \simeq \operatorname{Sym}^{\bullet, \star}(L[1]),
$$

and for $M=\left(\Delta^{\text {main }}\right)_{*}\left(M_{X}\right)$,

$$
\left(\Delta^{\text {main }}\right)^{!}\left(\operatorname{Sym}^{\bullet, \star}(M)\right) \simeq \operatorname{Sym}^{\bullet}, !\left(M_{X}\right) .
$$

Remark 6.5.4. Assume that $X$ is smooth of dimension $d$, and $L$ is such that $L[1-d]$ lies in the heart of the natural t-structure on $X$, and is flat as a quasi-coherent sheaf. Then Corollary 6.5.2 implies that $U^{\mathrm{ch}}(L)_{X}[1-d]$ also lies in the heart of the t-structure.

\section{6. ^-Factorization coalgebras.}


6.6.1. Let us denote by $\operatorname{Fact}^{\star}(X)$ the full subcategory of $\operatorname{Com}^{- \text {coalg }^{\star}}(\operatorname{Ran} X)$ equal to the preimage under

$$
\operatorname{oblv}_{\mathrm{Com}}^{\star \rightarrow \mathrm{ch}}: \operatorname{Com}-\operatorname{coalg}^{\star}(\operatorname{Ran} X) \rightarrow \text { Com-coalg }{ }^{\mathrm{ch}}(\operatorname{Ran} X)
$$

of the full subcategory Fact $(X) \subset \operatorname{Com}$-coalg ${ }^{\operatorname{ch}}(\operatorname{Ran} X)$.

We can encode Theorems 1.2.4 and 6.4.2 and Proposition 6.1.2 as the following commutative diagram:

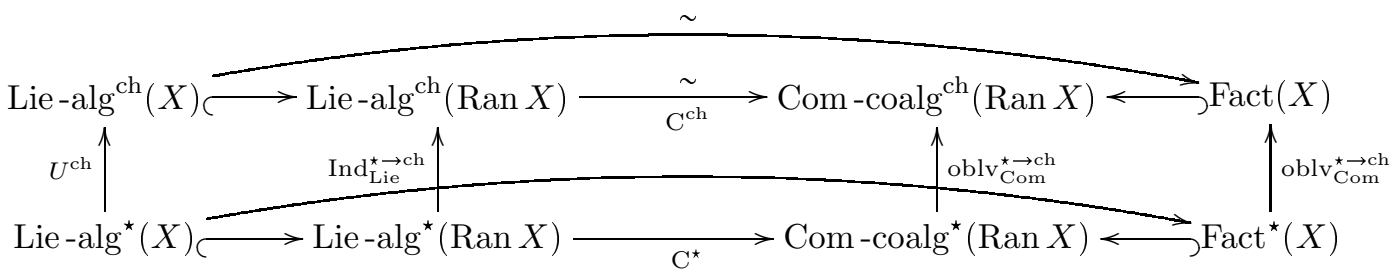

6.6.2. Note that, unlike $\mathrm{C}^{\mathrm{ch}}$, the functor

$$
\mathrm{C}^{\star}: \text { Lie }-\operatorname{alg}^{\star}(\operatorname{Ran} X) \rightarrow \text { Com }-\operatorname{coalg}{ }^{\star}(\operatorname{Ran} X)
$$

is not an equivalence, since the category $\mathfrak{D}(\operatorname{Ran} X)$ equipped with the $\star$ symmetric monoidal functor is not pro-nilpotent. For instance, for $X=(\mathrm{pt}):=\operatorname{Spec} k$, the above functor is the usual functor

$$
\mathrm{C}: \text { Lie-alg }\left(\operatorname{Vect}_{k}\right) \rightarrow \text { Com-coalg }\left(\text { Vect }_{k}\right),
$$

which is not an equivalence (since we include no nilpotence hypotheses on the algebras).

This example embeds into the case of any $X$ by choosing a $k$-point $x \in X$, and thus realizing Vect $_{k} \simeq \mathfrak{D}(\operatorname{Ran}(\mathrm{pt}))$ as a full subcategory of $\mathfrak{D}(\operatorname{Ran} X)$.

\section{Chiral And Factorization modules}

\subsection{Modules for algebras over an operad.}

7.1.1. We return to the setting of Section 3.2. Let $\mathcal{M}$ be a module $\infty$-category for $\mathcal{C}$. I.e., $\mathcal{M}$ is a $\mathcal{C}$-module in the symmetric monoidal $(\infty, 1)$-category of $\mathcal{X}$-modules in $\infty$-Cat pres,cont .

We can consider $\mathcal{M} \times \mathcal{C}$ as another symmetric monoidal $\infty$-category, where the monoidal operation on $\mathcal{M} \times 0_{\mathcal{C}}$ is zero. Let

$$
p: \mathcal{M} \times \mathcal{C} \longrightarrow \mathcal{C},(m \times c) \leadsto c
$$

denote the tautological homomorphism.

Definition 7.1.2. The $\infty$-category $\operatorname{Mod}_{A}(\mathcal{M})$ is the fiber of the functor

$$
p: \mathcal{O}-\operatorname{alg}(\mathcal{M} \times \mathcal{C}) \longrightarrow \mathcal{O}-\operatorname{alg}(\mathcal{C})
$$

over $A$.

The natural forgetful functor

$$
\operatorname{oblv}_{A}: \operatorname{Mod}_{A}(\mathcal{M}) \longrightarrow \mathcal{M} \times \mathcal{C} \longrightarrow \mathcal{M}
$$

admits a left adjoint, denoted Free ${ }_{A}$. The composition

$$
\mathrm{T}_{A}:=\text { Free }_{A} \circ \operatorname{oblv}_{A}: \mathcal{M} \longrightarrow \mathcal{M}
$$

is naturally a monad on $\mathcal{M}$, and by the Barr-Beck-Lurie theorem $\operatorname{Mod}_{A}(\mathcal{M}) \simeq \operatorname{Mod}_{\mathrm{T}_{A}}(\mathcal{M})$.

Similarly, for a cooperad $\mathcal{P}$ and $B \in \mathcal{P}$-coalg d.p. $_{\text {in }}^{\text {in }}(\mathcal{C})$, we introduce an $\infty$-category

$$
\operatorname{Comod}_{B}^{\text {nil }}(\mathcal{M})
$$

endowed with a forgetful functor $\operatorname{oblv}_{B}: \operatorname{Comod}_{B}^{\text {nil }}(\mathcal{M}) \rightarrow \mathcal{M}$, which admits a right adjoint

$$
\operatorname{coFree}_{B}: \mathcal{M} \rightarrow \operatorname{Comod}_{B}^{\text {nil }}(\mathcal{M}),
$$


so that

$$
\operatorname{Comod}_{B}^{\text {nil }}(\mathcal{M}) \simeq \operatorname{Comod}_{S_{B}}(\mathcal{M})
$$

where $\mathrm{S}_{B}:=\operatorname{oblv}_{B} \circ$ coFree $_{B}$.

It is easy to see from the construction that the $\infty$-categories $\operatorname{Mod}_{A}(\mathcal{M})$ and $\operatorname{Comod}_{B}^{\text {nil }}(\mathcal{M})$ are stable.

7.1.3. Let $\mathcal{O}$ and $A$ be as above. Set $\mathcal{O}^{\vee}:=\operatorname{Bar}(\mathcal{O})$ and $A^{\vee}:=\operatorname{Bar}_{\mathcal{O}}^{\text {enh }}(A)$. We have a tautological functor

$$
\operatorname{triv}_{A}: \mathcal{M} \longrightarrow \operatorname{Mod}_{A}(\mathcal{M})
$$

which commutes with limits and colimits. We denote by

$$
\operatorname{Bar}_{A}: \operatorname{Mod}_{A}(\mathcal{M}) \longrightarrow \mathcal{M}
$$

the left adjoint of $\operatorname{triv}_{A}$.

Lemma 7.1.4. The comonad $\operatorname{Bar}_{A} \circ \operatorname{triv}_{A}: \mathcal{M} \rightarrow \mathcal{M}$ is canonically equivalent to $\mathrm{S}_{A^{\vee}}$.

Hence, the functor $\operatorname{Bar}_{A}$ canonically upgrades to a functor

$$
\operatorname{Bar}_{A}^{\mathrm{enh}}: \operatorname{Mod}_{A}(\mathcal{M}) \longrightarrow \operatorname{Comod}_{A^{\vee}}^{\mathrm{nil}}(\mathcal{M})
$$

such that

$$
\operatorname{Bar}_{A} \simeq \operatorname{oblv}_{A \vee} \circ \operatorname{Bar}_{A}^{\mathrm{enh}}
$$

where oblv $A^{\vee}: \operatorname{Comod}_{A^{\vee}}^{\text {nil }}(\mathcal{M}) \rightarrow \mathcal{M}$ is the forgetful functor.

Definition 7.1.5. We shall say that a $\mathcal{C}$-module $\mathcal{M}$ is pro-nilpotent if $\mathcal{M}$ can be exhibited as

$$
\mathcal{M} \simeq \lim _{\mathbb{N o p}} \mathcal{M}_{i}
$$

(where the limit is taken in the $(\infty, 1)$-category of $\mathcal{C}$-modules), such that

- $\mathcal{M}_{0}=0$;

- For every $i \geqslant j$, the transition functor $f_{i, j}: \mathcal{M}_{i} \rightarrow \mathcal{M}_{j}$ commutes with limits;

- For every $i$, the restriction of the action functor $\mathcal{C} \otimes \mathcal{M}_{i} \rightarrow \mathcal{M}_{i}$ to $\mathcal{C} \otimes \operatorname{ker}\left(f_{i, i-1}\right)$ is nullhomotopic.

As in Proposition 4.1.2 one proves:

Proposition 7.1.6. Let $\mathcal{O} \in \mathrm{Op}(\mathcal{X})$ be an operad, and $A \in \mathcal{O}-\operatorname{alg}(\mathcal{C})$ an $\mathcal{O}$-algebra in $\mathcal{C}$, such that the adjunction $A \rightarrow \operatorname{Cobar}^{\mathcal{O}^{\vee}}\left(A^{\vee}\right)$ is a homotopy equivalence. Then for a pro-nilpotent $\mathcal{C}$-module $\mathcal{M}$, the functor (7.1)

$$
\operatorname{Bar}_{A}^{\mathrm{enh}}: \operatorname{Mod}_{A}(\mathcal{M}) \longrightarrow \operatorname{Comod}_{A^{\vee}}^{\mathrm{nil}}(\mathcal{M})
$$

is an equivalence.

7.1.7. Let us take $\mathcal{X}=\operatorname{Vect}_{k}$, where $\operatorname{char}(k)=0$, and $\mathcal{O}=$ Lie. From Proposition 7.1.6 and considerations analogous to those in Section 4.2 we obtain:

Corollary 7.1.8. Let $\mathcal{C}$ be pro-nilpotent, and let $\mathcal{M}$ be a pro-nilpotent $\mathcal{C}$-module. Then for $L \in$ Lie-alg $(\mathcal{C})$ and $B:=L^{\vee} \in$ Com-coalg $(\mathcal{C})$, the homological Chevalley complex functor

$$
\mathrm{C}_{L}: \operatorname{Mod}_{L}(\mathcal{M}) \longrightarrow \operatorname{Comod}_{B}(\mathcal{M})
$$

is an equivalence of $\infty$-categories.

7.2. Chiral and $\star$ actions. Let us take $\mathcal{C}$ to be $\mathfrak{D}(\operatorname{Ran} X)$ with either the $\star$ or the chiral symmetric monoidal structures. We shall now recall a class of $\mathfrak{D}(\operatorname{Ran} X)$-module $\infty$-categories for both structures. These categories we first introduced in $[\mathrm{Ro}]$. 
7.2.1. Let $I_{0}$ be a fixed finite set. Let $\mathrm{fSet}_{I_{0}}^{\mathrm{surj}}$ be the category of finite sets $I$ equipped with an arbitrary map $\pi_{0}: I_{0} \rightarrow I$, where the morphisms are maps under $I_{0}$ that are surjective.

As in Section 2.1 for a separated scheme $X$ we consider the functor

$$
X^{\left(\mathrm{fSet}_{I_{0}}^{\mathrm{surj}}\right)^{\mathrm{op}}} \longrightarrow \mathrm{Sch}:\left(I, \pi_{0}: I_{0} \rightarrow I\right) \leadsto X^{I},
$$

and the corresponding functor

$$
\mathfrak{D}^{!}\left(X^{\left(\mathrm{fSet}_{I_{0}}^{\mathrm{surj}}\right)^{\mathrm{op}}}\right): \mathrm{fSet}_{I_{0}}^{\text {surj }} \longrightarrow \infty-\mathrm{Cat}_{\text {pres,cont }}^{\mathrm{st}} .
$$

Definition 7.2.2. The $\infty$-category $\mathfrak{D}\left(\operatorname{Ran}_{I_{0}} X\right)$ is the limit of the functor in (17.2) in $\infty$-Cat pres,cont .

For $\left(I, \pi_{0}: I_{0} \rightarrow I\right)$ we let $\left(\Delta_{I_{0}}^{I}\right)^{!}$denote the tautological evaluation functor

$$
\mathfrak{D}\left(\operatorname{Ran}_{I_{0}} X\right) \longrightarrow \mathfrak{D}\left(X^{I}\right)
$$

For $I=I_{0}$ and $\pi_{0}=$ id, we will shall also use the notation $\left(\Delta_{I_{0}}^{\text {main }}\right)$ !.

As in Section 2.1 we have a canonical equivalence

$$
\mathfrak{D}\left(\operatorname{Ran}_{I_{0}} X\right) \simeq \operatorname{colim}_{\left(\operatorname{fSet}_{I_{0}}^{\text {surj }}\right)^{\text {op }}} \mathfrak{D}^{*}\left(X^{\mathrm{fSet}} \operatorname{Is}_{I_{0}}^{\text {surj }}\right) .
$$

For $\left(I, \pi_{0}: I_{0} \rightarrow I\right)$ we let $\left(\Delta_{I_{0}}^{I}\right)_{*}$ denote the resulting functor $\mathfrak{D}\left(\operatorname{Ran}_{I_{0}} X\right) \rightarrow \mathfrak{D}\left(X^{I}\right)$, which is easily seen to be the left adjoint of $\left(\Delta_{I_{0}}^{I}\right)$ !.

For $I=I_{0}$ and $\pi_{0}=\mathrm{id}$, we will shall also use the notation $\left(\Delta_{I_{0}}^{\text {main }}\right)_{*}$. It is easy to see that the adjunction map

$$
\mathrm{Id} \longrightarrow\left(\Delta_{I_{0}}^{\text {main }}\right)^{!} \circ\left(\Delta_{I_{0}}^{\text {main }}\right)_{*}
$$

is a homotopy equivalence, i.e., the functor $\left(\Delta_{I_{0}}^{\operatorname{main}}\right)_{*}$ is fully faithful.

Definition 7.2.3. We shall say that an object of $\mathfrak{D}\left(\operatorname{Ran}_{I_{0}} X\right)$ is supported on $X^{I_{0}}$ if it lies in the essential image of $\left(\Delta_{I_{0}}^{\text {main }}\right)_{*}$.

7.2.4. We shall now introduce the actions of $\mathfrak{D}(\operatorname{Ran} X)$ on $\mathfrak{D}\left(\operatorname{Ran}_{I_{0}} X\right)$ in the $\star$ and chiral contexts. We shall define the corresponding functors

$$
\mathfrak{D}(\operatorname{Ran} X)^{\otimes J} \otimes \mathfrak{D}\left(\operatorname{Ran}_{I_{0}} X\right) \longrightarrow \mathfrak{D}\left(\operatorname{Ran}_{I_{0}} X\right)
$$

in both contexts, in the style of Section 2.3 . Upgrading them to the actual datum of action is done as in Section 2.2

Using (2.3) and (7.3), to define a functor as in (7.4) it suffices to define a functor

$$
m_{J, I_{0}}: \underbrace{\left(\mathrm{fSet}^{\mathrm{surj}}\right)^{\mathrm{op}} \times \ldots \times\left(\mathrm{fSet}^{\mathrm{surj}}\right)^{\mathrm{op}}}_{J} \times\left(\mathrm{fSet}_{I_{0}}^{\mathrm{surj}}\right)^{\mathrm{op}} \longrightarrow\left(\mathrm{fSet}_{I_{0}}^{\text {surj }}\right)^{\mathrm{op}}
$$

and a natural transformation between the resulting two functors

$$
\begin{gathered}
\underbrace{\left(\mathrm{fSet}^{\text {surj }}\right)^{\mathrm{op}} \times \ldots \times\left(\mathrm{fSet}^{\mathrm{surj}}\right)^{\mathrm{op}}}_{J} \times\left(\mathrm{fSet}_{I_{0}}^{\mathrm{surj}}\right)^{\mathrm{op}} \rightrightarrows \infty-\mathrm{Cat}_{\text {pres }}^{\mathrm{st}}: \\
\left(\left(I_{J}, I_{0} \rightarrow I^{\prime}\right) \leadsto(\underbrace{\otimes}_{j \in J} \mathfrak{D}\left(X^{I_{j}}\right)) \otimes \mathfrak{D}\left(X^{I^{\prime}}\right)\right) \Rightarrow\left(\left(I_{J}, I_{0} \rightarrow I^{\prime}\right) \leadsto \mathfrak{D}\left(X^{m_{J, I_{0}}\left(I_{J}, I_{0} \rightarrow I^{\prime}\right)}\right)\right) .
\end{gathered}
$$

Here $I_{J}$ has the same meaning as in Section 2.2.3, and $I_{0} \rightarrow I^{\prime}$ is an object of fSet $I_{0}$. 
7.2.5. In both cases we set

$$
m_{J, I_{0}}\left(I_{J}, I_{0} \rightarrow I^{\prime}\right):=I \sqcup I^{\prime}
$$

where $I:=\underset{j \in J}{\sqcup} I_{j}$, with the map

$$
I_{0} \rightarrow I^{\prime} \hookrightarrow I \sqcup I^{\prime}
$$

We let $\pi$ denote the map $I \rightarrow J$ as in Section 2.2, Let $\pi_{I_{0}}$ denote the map $I \sqcup I^{\prime} \rightarrow J \sqcup$ pt that sends $I^{\prime}$ to pt.

For the $\star$ action, we define the functor of (7.5) to be the external tensor product

$$
\left(M^{I_{j}} \in \mathfrak{D}\left(X^{I_{j}}\right), M^{I^{\prime}} \in \mathfrak{D}\left(X^{I^{\prime}}\right)\right) \leadsto\left(\left(\underset{j}{\bigotimes} M^{I_{j}}\right) \bigotimes M^{I^{\prime}} \in \mathfrak{D}\left(X^{I \sqcup I^{\prime}}\right)\right) .
$$

For the chiral action we let the natural transformation (7.5) to be

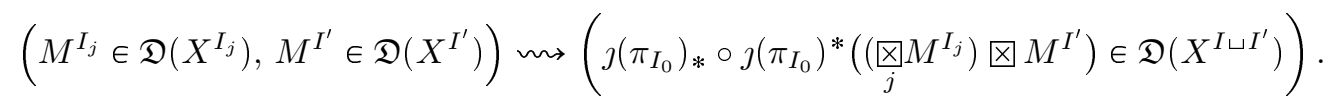

7.2.6. As in Lemma 2.3.4 for $M_{j} \in \mathfrak{D}(\operatorname{Ran} X), j \in J$ and $M^{\prime} \in \mathfrak{D}\left(\operatorname{Ran}_{I_{0}} X\right)$ we have an explicit description of the object

$$
\left(\underset{j \in J}{\otimes^{\mathrm{ch}}} M_{j}\right) \otimes{ }^{\mathrm{ch}} M^{\prime} \in \mathfrak{D}\left(\operatorname{Ran}_{I_{0}} X\right) .
$$

Namely, for $\left(I, \pi_{0}: I_{0} \rightarrow I\right) \in \operatorname{fSet}_{I_{0}}$ we have a canonical homotopy equivalence

$$
\left(\Delta_{I_{0}}^{I}\right)^{!}\left(\left(\underset{j \in J}{\otimes_{j \in J}^{\mathrm{ch}}} M_{j}\right) \otimes^{\mathrm{ch}} M^{\prime}\right) \simeq \underset{\pi_{\mathrm{pt}}}{\oplus} \jmath\left(\pi_{\mathrm{pt}}\right)_{*} \circ \jmath\left(\pi_{\mathrm{pt}}\right)^{*}\left(\left(\underset{j \in J}{\bigotimes}\left(\Delta^{I_{j}}\right)^{!}\left(M_{j}\right)\right) \otimes\left(\Delta_{I_{0}}^{I^{\prime}}\right)^{!}\left(M^{\prime}\right)\right),
$$

where the direct sum is taken over the set of all surjections $\pi_{\mathrm{pt}}: I \rightarrow J \sqcup \mathrm{pt}$, such that $\pi_{\mathrm{pt}} \circ \pi_{0}$ sends $I_{0} \rightarrow \mathrm{pt} \in J \sqcup \mathrm{pt}$, and where $I^{\prime} \subset I$ equals $\left(\pi_{\mathrm{pt}}\right)^{-1}(\mathrm{pt})$.

As in Section 5.1. the homotopy equivalence (7.6) implies that the chiral action of $\mathfrak{D}(\operatorname{Ran} X)$ on the module category $\mathfrak{D}\left(\operatorname{Ran}_{I_{0}} X\right)$ is nilpotent.

\subsection{Chiral and factorization modules.}

7.3.1. For $A \in \operatorname{Lie}-\operatorname{alg}^{\mathrm{ch}}(\operatorname{Ran} X)$, we let $\operatorname{Mod}_{A}^{\mathrm{ch}}\left(\operatorname{Ran}_{I_{0}} X\right)$ denote the resulting $\infty$-category of modules in $\mathfrak{D}\left(\operatorname{Ran}_{I_{0}} X\right)$. We call its objects chiral $A$-modules on $\operatorname{Ran}_{I_{0}} X$.

We shall denote by $\operatorname{Mod}_{A}^{\text {ch }}\left(X^{I_{0}}\right) \subset \operatorname{Mod}_{A}^{\text {ch }}\left(\operatorname{Ran}_{I_{0}} X\right)$ the full subcategory spanned by objects supported on $X^{I_{0}}$. We shall call its objects chiral $A$-modules on $X^{I_{0}}$.

Similarly, given $B \in$ Com-coalg $\left(\operatorname{Ran}_{X}\right)$, let $\operatorname{Comod}_{B}^{\text {ch }}\left(\operatorname{Ran}_{I_{0}} X\right)$ denote the $\infty$-category of $B$ comodules in $\mathfrak{D}\left(\operatorname{Ran}_{I_{0}} X\right)$. We shall call its objects chiral $B$-comodules on $\operatorname{Ran}_{I_{0}} X$.

From Theorem 5.1.1 and Corollary 7.1 .8 we obtain:

Corollary 7.3.2. For any $A \in \mathrm{Lie}-\operatorname{alg}^{\mathrm{ch}}(\operatorname{Ran} X)$, the functor

$$
\mathrm{C}_{A}^{\mathrm{ch}}: \operatorname{Mod}_{A}^{\mathrm{ch}}\left(\operatorname{Ran}_{I_{0}} X\right) \longrightarrow \operatorname{Comod}_{\mathrm{C}^{\mathrm{ch}}(A)}^{\mathrm{ch}}\left(\operatorname{Ran}_{I_{0}} X\right)
$$

is an equivalence. 
7.3.3. Let $B$ be an object of Com-coalg ${ }^{\text {ch }}(\operatorname{Ran} X)$, and let $N$ be an object of $\operatorname{Comod}_{B}^{\text {ch }}\left(\operatorname{Ran}_{I_{0}} X\right)$. For an object $\left(I, \pi_{0}: I_{0} \rightarrow I\right) \in \operatorname{fSet}_{I_{0}}$ and a map $\pi_{\mathrm{pt}}: I \rightarrow J \sqcup \mathrm{pt}$, from (7.6) we obtain a map

$$
\left(\Delta_{I_{0}}^{I}\right) !(N) \longrightarrow \jmath\left(\pi_{\mathrm{pt}}\right)_{*} \circ \jmath\left(\pi_{\mathrm{pt}}\right)^{*}\left(\left({\underset{j \in J}{\bigotimes}}_{j \in}\left(\Delta^{I_{j}}\right)^{!}(B)\right) \bigotimes\left(\Delta_{I_{0}}^{I^{\prime}}\right)^{!}(N)\right),
$$

and by adjunction a map

$$
\jmath\left(\pi_{\mathrm{pt}}\right)^{*}\left(\left(\Delta_{I_{0}}^{I}\right)^{!}(N)\right) \longrightarrow \jmath\left(\pi_{\mathrm{pt}}\right)^{*}\left(\left(\underset{j \in J}{\bigotimes}\left(\Delta^{I_{j}}\right)^{!}(B)\right) \bigotimes\left(\Delta_{I_{0}}^{I^{\prime}}\right)^{!}(N)\right) .
$$

Assume now that $B$ is a factorization coalgebra.

Definition 7.3.4. $N$ is a factorization B-comodule if, for every $\left(I, \pi_{0}: I_{0} \rightarrow I\right)$ and a map $\pi_{\mathrm{pt}}: I \rightarrow J \sqcup \mathrm{pt}$ as above, the map in (7.7) is a homotopy equivalence.

We let $\operatorname{Comod}_{B}^{\text {Fact }}\left(\operatorname{Ran}_{I_{0}} X\right)$ denote the full subcategory of $\operatorname{Comod}_{B}\left(\operatorname{Ran}_{I_{0}} X\right)$ spanned by factorization modules.

As in Section 5.2, one shows:

Corollary 7.3.5. Let $A$ be a chiral Lie algebra on X. The equivalence of Corollary 7.3.2 induces an equivalence between the subcategory $\operatorname{Mod}_{A}\left(X^{I_{0}}\right)$ of $\operatorname{Mod}_{A}\left(\operatorname{Ran}_{I_{0}} X\right)$ spanned by modules supported on $X^{I_{0}}$, and the subcategory of factorization $\mathrm{C}^{\mathrm{ch}}(A)$-comodules:

$$
\operatorname{Mod}_{A}\left(X^{I_{0}}\right) \simeq \operatorname{Comod}_{C^{\text {ch }}(A)}^{\mathrm{Fact}}\left(\operatorname{Ran}_{I_{0}} X\right)
$$

\section{REFERENCES}

[BD1] Beilinson, Alexander; Drinfeld, Vladimir. Chiral algebras. American Mathematical Society Colloquium Publications, 51. American Mathematical Society, Providence, RI, 2004. vi+375 pp.

[BD2] Beilinson, Alexander; Drinfeld, Vladimir. Quantization of Hitchin's integrable system and Hecke eigensheaves. Available from http://math.uchicago.edu/ mitya/langlands.html

[BFN] Ben-Zvi, David; Francis, John; Nadler, David. Integral transforms and Drinfeld centers in derived algebraic geometry. J. Amer. Math. Soc. 23 (2010), no. 4, 909966.

[BF] Frenkel, Edward; Ben-Zvi, David. Vertex algebras and algebraic curves. Second edition. Mathematical Surveys and Monographs, 88. American Mathematical Society, Providence, RI, 2004. xiv+400 pp.

[BN] Ben-Zvi, David; Nadler, David. The character theory of a complex group. Preprint, 2009. arXiv:0904.1247

[BFS] Bezrukavnikov, Roman; Finkelberg, Michael; Schechtman, Vadim. Factorizable sheaves and quantum groups. Lecture Notes in Mathematics 1691, Springer, 1998.

[BV] Boardman, J. Michael; Vogt, Rainer. Homotopy invariant algebraic structures on topological spaces. Lecture Notes in Mathematics, Vol. 347. Springer-Verlag, Berlin-New York, 1973. x+257 pp.

[C1] Ching, Michael. Bar constructions for topological operads and the Goodwillie derivatives of the identity. Geom. Topol. 9 (2005), 833933 (electronic).

[C2] Ching, Michael. Bar-cobar duality for operads in stable homotopy theory. Preprint, 2010. Available from http://www.math.uga.edu/ mching/publications.html

[CG] Costello, Kevin; Gwilliam, Owen. Factorization algebras in perturbative quantum field theory. Preprint.

[Dr] Drinfeld, Vladimir. DG quotients of DG categories. J. Algebra 272 (2004), no. 2, 643691.

[F1] Francis, John. Derived algebraic geometry over $\mathcal{E}_{n}$-rings. Thesis (PhD) Massachusetts Institute of Technology. 2008.

[F2] Francis, John. Factorization homology of topological manifolds. In preparation.

[Fr] Fresse, Benoit. On the homotopy of simplicial algebras over an operad. Trans. Amer. Math. Soc. 352 (2000), no. 9, 41134141.

[Ga1] Gaitsgory, Dennis. Twisted Whittaker model and factorizable sheaves. Selecta Math., New ser. 13 (2007), 617-659.

[Ga2] Gaitsgory, Dennis. Ind-coherent sheaves. Preprint, 2011. arXiv:1105.4857.

[GJ] Getzler, Ezra; Jones, Jon. Operads, homotopy algebra and iterated integrals for double loop spaces. Unpublished work, 1994.

[GK] Ginzburg, Victor; Kapranov, Mikhail. Koszul duality for operads. Duke Math. J. 76 (1994), no. 1, 203272.

[GL:DG] Notes on Geometric Langlands. DG Categories. Available from http://www.math.harvard.edu/ gaitsgde/GL/.

[Go] Goodwillie, Thomas. Calculus III: Taylor series. Geom. Topol. 7 (2003), 645711.

[Hi] Hinich, Vladimir DG coalgebras as formal stacks. J. Pure Appl. Algebra 162 (2001), no. 2-3, 209250. 
[Jo] Joyal, André. Quasi-categories and Kan complexes. Special volume celebrating the 70th birthday of Professor Max Kelly. J. Pure Appl. Algebra 175 (2002), no. 1-3, 207222.

[L1] Lurie, Jacob. Higher topos theory. Annals of Mathematics Studies, 170. Princeton University Press, Princeton, NJ, 2009. xviii+925 pp.

[L2] Lurie, Jacob. Higher Algebra. Prepublication book draft. Available from http://www.math.harvard.edu/ lurie/

[Mo] Moore, John. Differential homological algebra. Actes du Congrès International des Mathématiciens (Nice, 1970), Tome 1, Gauthier-Villars, Paris, 1971, pp. 335339.

[Q1] Quillen, Daniel. Homotopical algebra. Lecture Notes in Mathematics, No. 43 Springer-Verlag, Berlin-New York 1967 iv+156 pp.

[Q2] Quillen, Daniel. Rational homotopy theory. Ann. of Math. (2) 901969205295.

[Pr] Priddy, Stewart. Koszul resolutions, Trans. Amer. Math. Soc. 152 (1970), 3960.

[Ro] Rozenblyum, Nick. Modules over a chiral algebra. Preprint, 2010. arXiv:1010.1998

Department of Mathematics, Northwestern University, Evanston, IL 60208-2370

E-mail address: jnkf@math.northwestern.edu

Department of Mathematics, Harvard University, Cambridge, MA 02138-2901

E-mail address: gaitsgde@math.harvard.edu 